\title{
Sharp Results for the Regularity and Stability of the Free Boundary in the Obstacle Problem
}

\author{
Ivan Blank \\ Rutgers University \\ Piscataway, NJ 08854-8019 \\ blanki@math.rutgers.edu
}

November 2, 2003

\begin{abstract}
In this paper, we study the obstacle problem with obstacles whose Laplacians are not necessarily Hölder continuous. We show that the free boundary at a regular point is $C^{1}$ if the Laplacian of the obstacle is negative and Dini continuous. We also show that this condition is sharp by giving a method to construct a counter-example when we weaken the requirement on the Laplacian of the obstacle by allowing it to have any modulus of continuity which is not Dini. In the course of proving optimal regularity we also improve some of the perturbation theory due to Caffarelli (1981). Since our methods depend on comparison principles and regularity theory, and not on linearity, our stability results apply to a large class of obstacle problems with nonlinear elliptic operators.

In the case of obstacles where the Laplacian is negative and has sufficiently small oscillation, we establish measure-theoretic analogues of the alternative proven by Caffarelli (1977). Specifically, if the Laplacian is continuous, then at a free boundary point either the contact set has density zero, or the free boundary is a Reifenberg vanishing set and the contact set has density equal to one half in a neighborhood of the point. If the Laplacian is not necessarily continuous, but has sufficiently small oscillation, then at a free boundary point either the contact set has density close to zero, or the free boundary is a $\delta$-Reifenberg set and the contact set has density close to one half in a neighborhood of the point.
\end{abstract}




\section{Introduction}

Given a function $\varphi: B_{1} \rightarrow \mathbb{R}$ with $\varphi<0$ on $\partial B_{1}$ and $\max \varphi>0$, the least superharmonic function $u$ with $u \equiv 0$ on $\partial B_{1}$ and $u \geq \varphi$, is called the solution of the obstacle problem. $u$ is also the function which is the minimizer of the Dirichlet integral,

$$
\int_{B_{1}}\|\nabla u\|^{2}
$$

among all of the functions greater than or equal to $\varphi$, and equal to zero on the boundary of the ball. $\varphi$ is referred to as the obstacle, and $u$ is referred to as the membrane. The set $\{u=\varphi\}$ is called the contact set, and its boundary is called the free boundary. For $\varphi \in C^{\alpha}$ or $C^{1, \alpha}$, it is known that $u$ is as smooth as $\varphi$. (Originally due to Frehse, Lewy, and Stampacchia, and later extended and simplified by Brézis, Kinderlehrer, and Caffarelli. There are a variety of references listed in the bibliography.) On the other hand, no matter how smooth $\varphi$ is, $u$ will be no better than $C^{1,1}$. (Consider in $\mathbb{R}^{1}, \varphi(x):=1-3 x^{2}$. The second derivative of $u$ will jump from zero to -6 and back.)

The regularity of the boundary of the contact set, however, has more complicated behavior. First, in order to talk about the regularity of this free boundary, we need to make some sort of concavity assumption about $\varphi$. Without an assumption of this nature, we could solve the obstacle problem with a simple obstacle, and then a posteriori we could alter the obstacle to come up and touch our membrane on any closed set we liked. In the linear case, if the Laplacian of $\varphi$ is a Hölder continuous function bounded between two negative constants, Caffarelli has shown that any point on the free boundary is either in a $C^{1, \alpha}$ smooth portion of this boundary, or the contact set has to form a very thin "cusp-like" set (see [C1], [C2], and [C4]). So Lipschitz corners have been ruled out.

This paper is primarily concerned with what happens to this alternative when we weaken our assumptions on the smoothness of our obstacle. We show the following theorems:

0.1 Theorem ( Dini Laplacian Gives $\mathbf{C}^{\mathbf{1}}$ Regularity ). If the Laplacian of the obstacle is Dini continuous, and $x_{0}$ is a free boundary point in $B_{1 / 2}$ then there is a modulus of continuity, $\sigma(r)$, such that either

a. $x_{0}$ is a singular point of the free boundary and the minimum diameter of the contact set intersected with $B_{r}\left(x_{0}\right)$ is less than $r \sigma(r)$ for all $r>0$, or

b. $x_{0}$ is a regular point of the free boundary which is $C^{1}$ in a neighborhood of $x_{0}$.

(For the definition of minimum diameter, see Definition (3.1) below.) 
0.2 Theorem (Counter-Example). Let $\tilde{\sigma}$ be any modulus of continuity which is not Dini. In other words,

$$
\int_{0}^{\epsilon} \frac{\tilde{\sigma}(r)}{r} d r=+\infty
$$

for any $\epsilon>0$. No matter how slowly the Dini integral diverges, we can find an obstacle whose Laplacian has $\tilde{\sigma}$ as its modulus of continuity, and such that the contact set between the obstacle and membrane spins an infinite number of times around a point where this set has density $1 / 2$.

On the other hand, even if we don't assume continuity of the Laplacian of the obstacle, we can still distinguish between regular and singular points by measure theoretic considerations, and by concepts related to flatness, as long as the local oscillation of the Laplacian is sufficiently small. In the next two theorems we denote the contact set by $\Lambda$.

0.3 Theorem (Caffarelli's Alternative: Continuous Case). Assuming the Laplacian of the obstacle is continuous, and $x_{0}$ is a free boundary point in $B_{1 / 2}$, then either

a. $x_{0}$ is a singular point of the free boundary and

$$
\lim _{r \rightarrow 0} \frac{\left|\Lambda \cap B_{r}\left(x_{0}\right)\right|}{\left|B_{r}\right|}=0
$$

or

b. $x_{0}$ is a regular point of the free boundary and

$$
\lim _{r \rightarrow 0} \frac{\left|\Lambda \cap B_{r}\left(x_{0}\right)\right|}{\left|B_{r}\right|}=\frac{1}{2}
$$

for all $x$ in the free boundary which are sufficiently close to $x_{0}$. Furthermore, in this case, the free boundary is a Reifenberg vanishing set in a neighborhood of $x_{0}$.

(For the definition of Reifenberg flat and Reifenberg vanishing, see Definition (6.1) below.)

0.4 Theorem (Caffarelli's Alternative: Small Oscillation Case). If $\epsilon$ $>0$, there exists a $\delta>0$ such that if the Laplacian of the obstacle is within $\delta$ in $L^{\infty}$ norm of the constant function equal to one, and $x_{0}$ is a free boundary point in $B_{1 / 2}$ then either

a. $x_{0}$ is a singular point of the free boundary and

$$
\limsup _{r \rightarrow 0} \frac{\left|\Lambda \cap B_{r}\left(x_{0}\right)\right|}{\left|B_{r}\right|} \leq \epsilon,
$$

or 
b. $x_{0}$ is a regular point of the free boundary and

$$
\liminf _{r \rightarrow 0} \frac{\left|\Lambda \cap B_{r}\left(x_{0}\right)\right|}{\left|B_{r}\right|} \geq \frac{1}{2}-\epsilon
$$

for all $x$ in the free boundary which are sufficiently close to $x_{0}$. Furthermore, in this case, the free boundary is an $\epsilon-$ Reifenberg set in a neighborhood of $x_{0}$.

(For slightly more precise statements see Theorems (4.3), (4.5), and (6.4)). In particular, our counter-example is a case of a flat free boundary which is not Lipschitz. (It is not even locally the graph of a function.)

We attack this problem by means of a perturbation from the case where the Laplacian of the obstacle is constant. Roughly speaking, the idea is to surround the free boundary by smooth free boundaries of a sub and supersolution, and then to estimate the distance between the new free boundaries. In the process of carrying out this procedure we improve the uniform stability near regular points and the measure stability found in [C3]. In fact, even in the Hölder continuous case, without the improvements in the approximation theory, one cannot get the sharpest Hölder exponents in the free boundary regularity theory by the type of straightforward perturbation argument presented here. We also avoid the use of heavy machinery such as the Nash-Moser Implicit Function Theorem that Schaeffer used in [S1] and [S2], and we assume less regularity of our data and get stronger bounds than Stojanovic did in [St].

Finally, we note that our methods can be extended to some nonlinear obstacle problems without much effort. Assume that $F\left(D^{2} u(x), x\right)$ is uniformly elliptic and satisfies comparison (i.e. subsolutions of the Dirichlet problem

$$
F\left(D^{2} u(x), x\right)=0 \text { in } B_{1} \text { and } u=g \text { on } \partial B_{1}
$$

cannot be greater than the corresponding supersolutions), and $\varphi$ is a function satisfying:

1. $\varphi<0$ on $\partial B_{1}$,

2. $\max \varphi>0$, and

3. $F\left(D^{2} \varphi(x), x\right)<0$.

(A function satisfying the above requirements is called an obstacle.) Then the smallest supersolution of

$$
\begin{aligned}
F\left(D^{2} u(x), x\right)=0 & \text { in } \quad B_{1} \\
u=0 & \text { on } \partial B_{1}
\end{aligned}
$$


among functions which satisfy $u \geq \varphi$, is called the solution of the nonlinear obstacle problem. We will say that $F$ satisfies the Caffarelli Alternative if all obstacles $\varphi$ such that $F\left(D^{2} \varphi(x), x\right) \in C^{1}$ lead to solutions whose free boundaries satisfy the same alternative between regular and singular points (with different constants and different moduli of continuity), as the one given by Caffarelli for the linear case (see Theorems (3.2) and (3.4)). With these definitions we can now state our theorem for the nonlinear case.

0.5 Theorem (Dini Continuity Gives $\mathbf{C}^{1}$ Smoothness). If $F$ is a uniformly elliptic operator which

1. satisfies comparison and

2. satisfies the Caffarelli Alternative,

and $\varphi$ is an obstacle such that $F\left(D^{2} \varphi(x), x\right)$ is Dini continuous, then the free boundary of the corresponding solution to the nonlinear obstacle problem will be $C^{1}$ at regular points.

0.6 Remark. Recently Ki-ahm Lee has proven that the Caffarelli Alternative is satisfied by $F \in C^{1}$ which are homogeneous of degree 1 and which are either convex in any dimension, or concave in two dimensions (see $[\mathrm{L}]$ ). In particular, by using regularity results due to Brézis and Kinderlehrer, (see [BK]), we can apply this theorem to the minimal surface case.

0.7 Remark. Since the proofs in the nonlinear case do not differ substantially from the proofs in the linear case, we will only explicitly prove the linear versions of our theorems to simplify the exposition. For the nonlinear theorems we will only indicate the changes which would need to be made.

\section{Preliminaries}

For convenience we introduce the height function $w:=u-\varphi$. After this transformation, instead of minimizing the Dirichlet integral, we find that $w$ minimizes

$$
\int_{B_{1}}\left(\|\nabla w\|^{2}+2 g w\right),
$$

among nonnegative functions with the same boundary data, where we have defined $g=-\Delta \varphi$. Analysis of the Euler-Lagrange equation reveals the following situation: We have a set where $w \equiv 0$, and a set where $w>0$ and $\Delta w(x)=g(x)$. Since the Laplacian does not have a distribution across the interface of these two sets, we can conclude

$$
w \geq 0 \text { and } \Delta w(x)=\chi_{\{w>0\}} g(x) .
$$


We will always assume that $\psi, \varphi, f$, and $g$ are nonnegative functions. The domain of $\psi$ and $\varphi$ will be the boundary of a ball, and the domain of $f$ and $g$ will be the interior of a ball, or all of $\mathbb{R}^{n}$. We also fix $0<\lambda \leq f, g \leq \mu$, and to simplify the dependences of the constants, we assume always that $\lambda \in[1 / 2,3 / 2]$.

The following will be very important notation:

1. For any solution, $w$, to an obstacle problem, we define

$$
\begin{aligned}
& \Omega(w):=\{w>0\}, \\
& \Lambda(w):=\{w=0\}, \text { and } \\
& F B(w):=\partial \Omega(w) \cap \partial \Lambda(w) .
\end{aligned}
$$

2. $O P_{R}(\mathrm{Lap}=g, \mathrm{Bdry}=\psi)$ will denote the minimizer of

$$
\int_{B_{R}}\left(\|\nabla w(x)\|^{2}+2 g(x) w(x)\right) d x
$$

among nonnegative functions, $w \in H^{1}\left(B_{1}\right)$, whose trace on the boundary of $B_{R}$ is equal to $\psi$ (so $w$ satisfies equation (1.1)). When $R=1$ we will omit it. All of these functions will be referred to as solutions of the obstacle problem. If we have a function $W$ defined on $\mathbb{R}^{n}$ such that its restriction to any ball is a solution of the obstacle problem with $g \equiv c$, then we will write $W \in O P_{\infty}(\operatorname{Lap}=c)$.

3. $\mathcal{P}_{R}(\lambda, \mu, B)$ will denote the set of all functions $w$ which are of the form $w=O P_{R}\left(\right.$ Lap $=g$, Bdry $\left.=\left.w\right|_{B_{R}}\right)$ where $\lambda \leq g \leq \mu, g \in B$, and $0 \in$ $F B(w)$. $B$ will be either $L^{\infty}$ or a Banach space of continuous functions. $\widehat{\mathcal{P}}_{R}(\lambda, \mu, B)$ will denote the subset of $\mathcal{P}_{R}(\lambda, \mu, B)$ whose elements have zero sets, $\Lambda$, with density greater than $1 / 4$ at zero. Because of its particularly important role, we will use $\mathcal{P}_{R}(c)$ to denote the set of functions, $w$, of the form $w=O P_{R}\left(\operatorname{Lap}=c\right.$, Bdry $\left.=\left.w\right|_{B_{R}}\right)$ with $0 \in$ $F B(w)$. In all cases, when $R=1$ we will omit it.

We also make the following notational conventions:

1. For $S, T \subset \mathbb{R}^{n}$ we define

$$
S+T:=\{x: x=s+t \text { for some } s \in S \text { and } t \in T\} .
$$

So in particular $S+B_{r}$ is an $r$ neighborhood of the set $S$.

2. We will denote the ball of radius $r$ in $\mathbb{R}^{n-1}$ with a prime. (So $B_{r}^{\prime}=\left\{x^{\prime} \in\right.$ $\left.\mathbb{R}^{n-1}: \| x^{\prime}||<r\right\}$.) 
1.1 Remark. The minimizer $O P_{R}(\operatorname{Lap}=g$, Bdry= $\psi)$ will always exist for reasonable $g$ and $\psi$ since we are solving a minimization problem on a convex set in Hilbert space.

1.2 Remark. Suppose $w \in \mathcal{P}\left(\lambda, \mu, L^{\infty}\right)$ and $s \in(0,1)$. Then the rescaling:

$$
w_{s}(x):=s^{-2} w(s x)
$$

satisfies $w_{s} \in \mathcal{P}_{1 / s}\left(\lambda, \mu, L^{\infty}\right)$ and in particular $\left.w_{s}\right|_{B_{1}} \in \mathcal{P}\left(\lambda, \mu, L^{\infty}\right)$. Also, the same statements hold in $\widehat{\mathcal{P}}_{R}\left(\lambda, \mu, L^{\infty}\right)$ and in $\mathcal{P}_{R}\left(\lambda, \mu, C\left(\mathbb{R}^{n}\right)\right)$.

1.3 Remark. We can also look at the height function for the fully nonlinear obstacle problem, and basically the following problems are equivalent for most intents and purposes (including existence, uniqueness, and free boundary regularity) given the "right" assumptions, which we state below.

1. Finding a $u \in W_{l o c}^{2, p}\left(B_{1}\right) \cap C\left(\bar{B}_{1}\right)$ for all $p \in[1, \infty)$ with $u \equiv 0$ on $\partial B_{1}$ such that within $B_{1}, u$ satisfies:

$$
F\left(D^{2} u(x), x\right) \leq 0, u \geq \phi, \text { and }(u(x)-\phi(x)) F\left(D^{2} u(x), x\right)=0 .
$$

2. Finding a $w \in W_{l o c}^{2, p}\left(B_{1}\right) \cap C\left(\bar{B}_{1}\right)$ for all $p \in[1, \infty)$ with $w=\psi$ on $\partial B_{1}$ such that within $B_{1}, w$ satisfies:

$$
w \geq 0 \text { and } G\left(D^{2} w(x), x\right)=f(x) \text { in }\{w>0\} .
$$

We will say

$$
w=O P\left(G\left(D^{2} w\right)=f, w=\psi \text { on } \partial B_{1}\right) .
$$

The right assumptions to make are that $f(x)$ and $-F\left(D^{2} \phi(x), x\right)$ are positive and have the same regularity, that $F$ and $G$ have the same regularity, that $|F(0, x)| \leq C$ and $G(0, x)=0$, and that $F$ and $G$ are both convex, both concave, or neither.

\section{Basic Results and Comparison Theorems}

2.1 Theorem (Non-Degeneracy). Let $w \in \mathcal{P}\left(\lambda, \mu, L^{\infty}\right)$ and $x_{0} \in \bar{\Omega}$. Suppose $\overline{B_{r}\left(x_{0}\right)} \subset B_{1}(0)$, then

$$
\underset{\frac{\sup }{B_{r}\left(x_{0}\right)}}{ } w \geq \frac{\lambda}{2 n} r^{2}+w\left(x_{0}\right) \geq \frac{\lambda}{2 n} r^{2} .
$$


Proof. By continuity we can take $x_{0} \in \Omega$. Now let

$$
u(x):=w(x)-\frac{\lambda}{2 n}\left|x-x_{0}\right|^{2} \quad \text { in } \quad D:=\Omega \cap B_{r}\left(x_{0}\right) .
$$

Since $\Delta u \geq 0$ in $D$, the maximum of $u$ on $\bar{D}$ occurs on $\partial D$. But $u\left(x_{0}\right)=$ $w\left(x_{0}\right)>0$, so this maximum is positive. On the other hand, on $\partial \Omega$ we have $u(x)=-\frac{\lambda}{2 n}\left|x-x_{0}\right|^{2}<0$. Thus, there exists an $\tilde{x} \in\left\{\partial B_{r}\left(x_{0}\right)\right\} \cap \Omega \subset \partial D$ with $w(\tilde{x}) \geq \frac{\lambda}{2 n} r^{2}+w\left(x_{0}\right) \geq \frac{\lambda}{2 n} r^{2}$.

Q.E.D.

Note that we did not use the fact that $0 \in F B(w)$. One of the most important uses of this theorem will be to eventually give us a sort of "free-boundary compactness" property of quadratic rescalings of elements of $\widehat{\mathcal{P}}\left(\lambda, \mu, L^{\infty}\right)$. The nonlinear obstacle problem analogue is proven in $[\mathrm{L}]$.

Now we define a simple auxiliary function which we will use often:

$$
\Phi(x):=\frac{1-\|x\|^{2}}{2 n},
$$

and note:

$$
\begin{aligned}
& \Delta \Phi \equiv-1,\left.\quad \Phi\right|_{\partial B_{1}} \equiv 0, \\
& \text { and } 0 \leq \Phi \leq \frac{1}{2 n} \text { in } B_{1} .
\end{aligned}
$$

2.2 Lemma (The Bound on $\left.\mathbf{B}_{\mathbf{1 / 2}}\right)$. Let $w \in \mathcal{P}\left(\lambda, \mu, L^{\infty}\right)$. Then

$$
\left.w\right|_{B_{1 / 2}} \leq C(n) \mu .
$$

Proof. Let $w=O P(\operatorname{Lap}=f, \operatorname{Bdry}=\psi)$, where $\lambda \leq f \leq \mu$ as always. Now set $w_{1}=0$ on $\partial B_{1}$, and $\Delta w_{1}=\chi_{\{w>0\}} f \leq \mu$ in $B_{1}$. $w_{1}<0$ in $B_{1}$ since $\Delta w_{1} \geq 0$. But $\Delta\left(w_{1}+\mu \Phi\right) \leq \mu-\mu=0$, so $w_{1}+\mu \Phi \geq 0$ and thus $-\frac{\mu}{2 n} \leq-\mu \Phi \leq w_{1}<0$.

Let $w_{2}=w$ on $\partial B_{1}$, and $\Delta w_{2}=0$ in $B_{1}$. Then $w_{2}>0$ in $B_{1}$. Also $w_{1}+w_{2}=w$. Since $w(0)=0(w \in \mathcal{P}), w_{2}(0)=-w_{1}(0) \leq \frac{\mu}{2 n}$, and Harnack's inequality implies $\left.w_{2}\right|_{B_{1 / 2}} \leq C(n) \mu$.

Q.E.D.

2.3 Theorem (Regularity). For any $\alpha \in(0,1)$, there exists a constant $C=C(n, \alpha)$ such that if $w \in \mathcal{P}\left(\lambda, \mu, L^{\infty}\right)$, then

$$
\|w\|_{C^{1, \alpha}\left(\overline{B_{1}}\right)} \leq C(n, \alpha) \mu .
$$

Proof. Cordes-Nirenberg estimates and the lemma above yield this result immediately.

Q.E.D. 
2.4 Theorem (The Parabolic Bound). If $w \in \mathcal{P}\left(\lambda, \mu, L^{\infty}\right)$, then

$$
w(x) \leq C(n) \mu\|x\|^{2} \text { in } B_{1 / 2} .
$$

Proof. If not, then by using our rescaling (see equation (1.2)) we will contradict the last lemma.

Q.E.D.

For the proof of the last few regularity results we have essentially redone the proof from $[\mathrm{CK}]$, but in our variable $w$ (instead of with $u$ and $\varphi$ ). Again, the analogous results for the nonlinear problem are proven in [L].

Although we can only expect a bound of $C r^{1+\alpha}$ from our regularity result, we get an optimal bound of $C r^{2}$ from our rescaling combined with the lemma above. This bound is essential to get the compactness we need. It means that we can do a quadratic "blow-up" and our Cordes-Nirenberg estimates won't degenerate. Thus, we will have compactness in $C^{1, \alpha}$ by Arzela-Ascoli. We summarize this information in the next corollary.

2.5 Corollary (Existence of a "Blow-Up"). If $w \in \mathcal{P}\left(\lambda, \mu, L^{\infty}\right)$, then there exists a $\tilde{w} \in C^{1, \alpha}$, and a sequence $\left\{s_{k}\right\} \rightarrow 0$ such that $w_{s_{k}} \rightarrow \tilde{w}$, and $\nabla w_{s_{k}} \rightarrow \nabla \tilde{w}$ uniformly on $\bar{B}_{1}$.

To prove the next corollary, simply integrate in an appropriate direction, and use the fact that the gradients can't turn faster than the Hölder norm allows.

\subsection{Corollary (Basic Interpolation Estimate).}

Suppose

$$
\left\|w_{i}\right\|_{C^{1, \alpha}\left(\overline{B_{1}}\right)} \leq C_{0}(n, \alpha) \mu \text { and }\left\|w_{1}-w_{2}\right\|_{C^{0}\left(\overline{B_{1}}\right)} \leq \epsilon .
$$

Then

$$
\left\|\nabla w_{1}-\nabla w_{2}\right\|_{C^{0}\left(\overline{B_{1-\epsilon}}\right)} \leq C_{1}(n, \alpha) \mu \epsilon^{\frac{\alpha}{\alpha+1}} .
$$

\subsection{Theorem (Comparison Principles).}

a. Let $\psi_{1} \leq \psi_{2} \leq \psi_{1}+\epsilon$, and let $w_{i}:=O P\left(\right.$ Lap $\left.=g, B d r y=\psi_{i}\right)$. Then

$$
w_{1} \leq w_{2} \leq w_{1}+\epsilon .
$$

In particular,

$$
\left\|w_{1}-w_{2}\right\|_{\infty} \leq\left\|\psi_{1}-\psi_{2}\right\|_{\infty} .
$$

b. Let $g_{1} \leq g_{2} \leq g_{1}+\epsilon$, and let $w_{i}:=O P\left(L a p=g_{i}, B d r y=\psi\right)$. Then

$$
w_{2}(x) \leq w_{1}(x) \leq w_{2}(x)+\frac{\epsilon}{2 n}\left(1-\|x\|^{2}\right) .
$$


c. Let $w_{i}:=O P\left(L a p=g_{i}, B d r y=\psi\right)$. Then we get:

$$
\left\|w_{1}-w_{2}\right\|_{\infty} \leq C(n)\left\|g_{1}-g_{2}\right\|_{L^{n}\left(B_{1}\right)} .
$$

d. Let $w_{i}:=O P\left(\operatorname{Lap}=g_{i}, B d r y=\psi_{i}\right)$, with $g_{1} \leq g_{2}$, and $\psi_{1} \geq \psi_{2}$. Then

$$
\begin{aligned}
& w_{2} \leq w_{1}, \text { and in particular } \\
& \Lambda\left(w_{1}\right) \subset \Lambda\left(w_{2}\right) \text { and } \Omega\left(w_{2}\right) \subset \Omega\left(w_{1}\right) .
\end{aligned}
$$

Proof. All of these proofs are trivial applications of the maximum principle.

a. Let $u:=w_{2}-w_{1}$ and suppose $u$ has a negative minimum at $x_{0}$. Then $w_{1}\left(x_{0}\right)>0$, and so $\Delta u\left(x_{0}\right)=\Delta w_{2}\left(x_{0}\right)-g\left(x_{0}\right) \leq 0$, which is impossible, therefore $w_{1} \leq w_{2}$. Now let $w_{3}:=O P\left(\operatorname{Lap}=g, \quad\right.$ Bdry $\left.=\psi_{1}+\epsilon\right)$. Then $w_{1} \leq w_{2} \leq w_{3}$.

Now let $v:=w_{1}+\epsilon-w_{3}$, and suppose that $v$ has a negative minimum at $x_{0}$. Then $w_{3}>0$, and so $\Delta v\left(x_{0}\right)=\Delta w_{1}\left(x_{0}\right)-g\left(x_{0}\right) \leq 0$, which is impossible, so $w_{3} \leq w_{1}+\epsilon$.

b. It suffices to assume that $g_{2}=g_{1}+\epsilon$. We recall our auxiliary function $\Phi(x):=\frac{1}{2 n}\left(1-|x|^{2}\right)$ from $(2.2)$, and define $u:=w_{1}+\epsilon \Phi-w_{2}$. Suppose that $u$ has a negative minimum at $x_{0}$. Then $w_{2}\left(x_{0}\right)>0$, and therefore $\Delta u\left(x_{0}\right) \leq g_{1}\left(x_{0}\right)-\epsilon-g_{2}\left(x_{0}\right)<0$, which is impossible, so $w_{1}+\epsilon \Phi \geq w_{2}$.

c. Here the proof is the same as the last one, but instead of using $\epsilon \Phi$, we use the function $\Theta$, defined to be the solution of the following Dirichlet problem:

$$
\Delta \Theta(x)=-\left|g_{1}(x)-g_{2}(x)\right| \text { in } B_{1} \text {, and } \Theta(x) \equiv 0 \text { on } \partial B_{1} \text {. }
$$

d. Apply the maximum principle as above to $w_{1}-w_{2}$.

Q.E.D.

2.8 Corollary (Uniqueness). If $0<\lambda \leq f \leq \mu$, and $\psi$ is nonnegative, then $O P(\operatorname{Lap}=f, B d r y=\psi)$ is unique.

2.9 Remark. By combining our comparison principles above with Corollary (2.6) we can get estimates on the supremum norm of the difference of the gradients of two solutions to the obstacle problem. 
Our comparison principles also allow us to gain much more information about the blow-up we produced in Corollary (2.5). To state this result in its full generality we need to introduce a new class of functions. Let

$$
\begin{array}{r}
V_{n}(0):=\left\{f \in L^{\infty}\left|f(0):=\lim _{r \rightarrow 0} f_{B_{r}}\right| f(x) \mid d x\right. \text { exists, and } \\
\left.\lim _{r \rightarrow 0}\left(f_{B_{r}}|f(x)-f(0)|^{n} d x\right)=0\right\} .
\end{array}
$$

2.10 Theorem (Properties of the "Blow-Up"). If $w \in \mathcal{P}\left(\lambda, \mu, V_{n}(0)\right)$, then there exists a $\tilde{w} \in C^{1, \alpha}$, and a sequence $\left\{s_{k}\right\} \rightarrow 0$ such that $w_{s_{k}} \rightarrow \tilde{w}$, and $\nabla w_{s_{k}} \rightarrow \nabla \tilde{w}$ uniformly on $\bar{B}_{1}$.

Furthermore

a. $\tilde{w}=O P\left(\operatorname{Lap}=f(0), \quad B d r y=\left.\tilde{w}\right|_{B_{1}}\right)$ (so $\tilde{w}$ is a solution to the obstacle problem).

b. $\tilde{w}$ is the restriction an element in $O P_{\infty}(\operatorname{Lap}=f(0))$.

c. If $w \in \widehat{\mathcal{P}}\left(\lambda, \mu, V_{n}(0)\right)$, then in an appropriate system of coordinates $\tilde{w}(x)=\frac{1}{2} f(0)\left(x_{n}^{+}\right)^{2}$. (Note that the system of coordinates may depend on the sequence $\left\{s_{k}\right\}$ which is not necessarily unique.)

d. If $w \in \widehat{\mathcal{P}}\left(\lambda, \mu, V_{n}(0)\right)$, then $\partial \Lambda\left(w_{s_{k}}\right)$ converges uniformly to $\left\{x_{n} \equiv 0\right\}$ on compact sets.

Proof. The first part of this theorem is just a weaker version of Corollary (2.5) (weaker since $V_{n}(0) \subset L^{\infty}$ ).

a. This part follows from the uniform convergence of $w_{s_{k}}$ to $\tilde{w}$ on $\partial B_{1}$ combined with Theorem (2.7)a, Theorem (2.7)c, and our definition of $V_{n}(0)$.

b. This part follows by taking subsequences of the $w_{s_{k}}$ to get $\tilde{w}_{R}$ defined on larger and larger balls and then letting $\tilde{w}(x):=\lim _{R \rightarrow \infty} \tilde{w}_{R}(x)$ outside of $B_{1}$.

We postpone the proofs of parts c and d until the end of the next section. 


\section{Further Known Results}

Now we need to state a variety of theorems which have already been proven. Although we will be studying the solutions to obstacle problems with Laplacians which are either continuous or $L^{\infty}$ in the positivity set, it will suffice to state the results in this section for solutions to obstacle problems with Laplacians which are constant on their set of positivity. A good recent reference for most of the material in this section for the linear case is [C4]. The reference for the nonlinear versions is $[\mathrm{L}]$. In the nonlinear case Caffarelli's Alternative has been proven for convex operators in any dimension, and for concave operators in $\mathbb{R}^{2}$. Now we need to make a definition which will allow us to measure the "flatness" of a set.

3.1 Definition (Minimum Diameter). Given a set $S \in \mathbb{R}^{n}$, we define the minimum diameter of $S$ (or m.d.(S) ) to be the infimum among the distances between pairs of parallel hyperplanes enclosing $S$.

3.2 Theorem (Caffarelli's Alternative). There exists a modulus of continuity $\sigma(\rho)$ depending only on $n$ such that if $\lambda \leq c \leq \mu, 0 \in F B(w)$, and $w=O P($ Lap $=c, B d r y=\psi)$, then either

a. 0 is a Singular Point of $F B(w)$ :

m.d. $\left(\Lambda \cap B_{\rho}\right) \leq \rho \sigma(\rho)$, for all $\rho \leq 1$, or

b. 0 is a Regular Point of $F B(w)$ :

there exists a $\rho_{0}$ such that m.d. $\left(\Lambda \cap B_{\rho_{0}}\right) \geq \rho_{0} \sigma\left(\rho_{0}\right)$, and for all $\rho<$ $\rho_{0}, \operatorname{m.d} .\left(\Lambda \cap B_{\rho}\right) \geq C \rho \sigma\left(\rho_{0}\right)$.

Furthermore, in the case that 0 is regular, there exists a $\rho_{1}$ such that for any $x \in B_{\rho_{1}} \cap \partial \Omega(w)$, and any $\rho<2 \rho_{1}$, we have

$$
\begin{aligned}
& m . d .\left(\Lambda \cap B_{\rho}(x)\right) \geq C \rho \sigma\left(2 \rho_{1}\right), \text { and thus } \\
& \frac{\Lambda \cap B_{\rho}(x)}{B_{\rho}(x)} \geq C \sigma\left(2 \rho_{1}\right)>0 .
\end{aligned}
$$

So the set of regular points is an open subset of the free boundary, and at any singular point the zero set must become "cusp-like." Examples of solutions with singular points can be found in [S3], and in [C4] Caffarelli has shown that these singular points must lie in a $C^{1}$ manifold. The theorem above can be found in [C1] or [C2].

3.3 Theorem (Behavior Near a Regular Point). Given $\rho>0$, there exists an $\epsilon=\epsilon(\rho)$ and an $M=M(\rho)$, such that if $w \in \widehat{\mathcal{P}}_{M}(1)$, and m.d. $(\Lambda(w) \cap$ $\left.B_{1}\right)>2 n \rho$, then in an appropriate system of coordinates the following are satisfied for $x$ such that $\left|x^{\prime}\right|<\rho / 16$ and $-1<x_{n}<1$, and any unit vector $\tau$ with $\tau_{n}>0$ and $\left\|\tau^{\prime}\right\| \leq \rho / 16$ : 
a. $D_{\tau} w \geq 0$.

b. All level surfaces $\{w=c\}, c>0$, are Lipschitz graphs:

$$
x_{n}=f\left(x^{\prime}, c\right) \text { with }\|f\|_{L i p} \leq \frac{C(n)}{\rho} \text {. }
$$

c. $D_{e_{n}} w(x) \geq C(\rho) d(x, \Lambda)$.

d. For $\left\|\tau^{\prime}\right\| \leq \rho / 32, D_{\tau} w \geq C(\rho) d(x, \Lambda)$.

This last theorem summarizes results due to Caffarelli and Alt (see [C1] and $[\mathrm{A}]$ ). If we apply the theory of harmonic functions in Lipschitz domains (see $[\mathrm{AC}],[\mathrm{CFMS}]$, and $[\mathrm{JK}]$ ), then we can deduce the following result found in $[\mathrm{C} 4]$.

3.4 Theorem ( $\mathbf{C}^{1, \alpha}$ Regularity of Regular Points). Suppose that $w \in$ $\mathcal{P}(1)$. There exists a universal modulus of continuity $\sigma(\rho)$ such that if for one value of $\rho$, say $\rho_{0}, \operatorname{m.d} .\left(\Lambda \cap B_{\rho_{0}}\right)>\rho_{0} \sigma\left(\rho_{0}\right)$, then in a $\rho_{0}^{2}$ neighborhood of the origin, the free boundary is a $C^{1, \alpha}$ surface $x_{n}=f\left(x^{\prime}\right)$ with

$$
\|f\|_{C^{1, \alpha}} \leq \frac{C(n)}{\rho_{0}}
$$

3.5 Remark. Note that by the last theorem, the $C^{1, \alpha}$ norm of the free boundary will decay in a universal way at any regular point under our standard rescaling if we are allowed to rotate the coordinates.

3.6 Theorem (Measure Stability). Let $0 \leq \psi_{2} \leq \psi_{1} \leq \psi_{2}+c_{2}-c_{1}$, $0<\lambda \leq c_{1} \leq c_{2} \leq \mu, \quad$ and $\quad w_{i}:=O P\left(\operatorname{Lap}=c_{i}\right.$, Bdry $\left.=\psi_{i}\right)$. Then

$$
\mathcal{L}^{n}\left(\Lambda\left(w_{2}\right) \Delta \Lambda\left(w_{1}\right)\right)=\mathcal{L}^{n}\left(\Omega\left(w_{2}\right) \Delta \Omega\left(w_{1}\right)\right) \leq C(n, \mu) \sqrt{c_{2}-c_{1}} .
$$

3.7 Theorem (Finite Hausdorff Measure). $w=O P(\operatorname{Lap}=\mu, B d r y=\psi)$ implies

$$
\mathcal{H}^{n-1}\left(F B(w) \cap B_{1 / 2}\right) \leq C(n)
$$

The last two theorems can be found in [C3]. The last theorem is proven in [C3] in the case where the Laplacian of $w$ in the positivity set is a positive Lipschitz function, and in fact, all that is required in that argument is that the Laplacian of $w$ be in $W^{1, p}$ for some $p>n$. Finally we mention the following result due to Kinderlehrer and Nirenberg (see $[\mathrm{KN}]$ ).

3.8 Theorem (Higher Regularity). If $w \in O P(\operatorname{Lap}=f, B d r y=\psi)$ and 0 is a regular point of $F B(w)$, then $f \in C^{m, \alpha}\left(B_{1}\right)$ implies $F B(w)$ is a hypersurface of class $C^{m+1, \alpha}$ in some neighborhood of 0 . Furthermore, if $f$ is analytic, then (near the origin) so is $F B(w)$. 


\section{Proof of Theorem (2.10), continued.}

c. Clearly $0 \in \Lambda$. The fact that 0 is a regular point of $F B(\tilde{w})$ follows from our Nondegeneracy Theorem $(2.1)$, and the fact that $F B(\tilde{w})$ is a hyperplane follows from Theorem (3.4) above. Now by reflection we get $\tilde{w}(x)=\frac{1}{2} f(0) d(x, \Lambda)^{2}$ immediately.

d. By rescaling, it suffices to prove this result on $B_{1}$. By the uniform convergence of $\nabla w_{s_{k}}$ to $\nabla \tilde{w}$, and by Theorem (3.3)d we get for sufficiently large $k$ that

$$
D_{\tau} w_{s_{k}} \geq C d(x, \Lambda(\tilde{w}))-\epsilon \geq-\epsilon .
$$

Since $\nabla w_{s_{k}} \equiv 0$ in $\Lambda\left(w_{s_{k}}\right)$, for any $x \in \Lambda\left(w_{s_{k}}\right)$ we have

$$
d(x, \Lambda(\tilde{w})) \leq \frac{\epsilon}{C} .
$$

Thus, $\Lambda\left(w_{s_{k}}\right) \subset \Lambda(\tilde{w})+B \frac{\epsilon}{C}$.

On the other hand, if we have an $x \in \Omega\left(w_{s_{k}}\right)$ with $d(x, \Lambda(\tilde{w})) \geq \epsilon$ then by our Nondegeneracy Theorem (2.1) we get an $x_{0} \in B_{\epsilon}(x)$ with $w_{s_{k}}\left(x_{0}\right) \geq C \epsilon^{2}$. So, by the uniform convergence of $w_{s_{k}}$ to $\tilde{w}$, if $k$ was chosen sufficiently large, then $x_{0} \in \Omega(\tilde{w})$. Thus, $\Omega\left(w_{s_{k}}\right) \subset\left\{\Omega(\tilde{w})+B_{\epsilon}\right\}$. Putting things together, we get

$$
\begin{aligned}
F B\left(w_{s_{k}}\right) & \subset\left\{\Lambda(\tilde{w})+B_{\frac{\epsilon}{C}}\right\} \cap\left\{\Omega(\tilde{w})+B_{\epsilon}\right\} \\
& \subset F B(\tilde{w})+B_{C \epsilon} .
\end{aligned}
$$

Q.E.D.

3.9 Remark. Our results above imply that at regular points of $F B(\tilde{w})$ the density of $\Lambda(\tilde{w})$ is $\frac{1}{2}$, and at singular points of $F B(\tilde{w})$ the density of $\Lambda(\tilde{w})$ is 0 .

\section{Measure Stability Results}

In this section we will extend and improve the measure stability result (Theorem (3.6) ) which was proven by Caffarelli in [C3], and give some applications of our improvements. Our main extension will be to deal with obstacle problems where the Laplacian does not have to be Hölder continuous. The main improvement will be to have the measure of the nonoverlapping regions of the zero set bounded by a constant times the change in the Laplacian instead of being bounded by the square root of the change in the Laplacian. Results of this type were obtained by Schaeffer in [S1] and [S2], although he used the Nash-Moser Implicit Function Theorem. Our proof will be much more elementary. 
4.1 Theorem (Improved Measure Stability). Assume $0<\lambda \leq g_{i} \leq \mu$, and set $v_{i}:=O P\left(L a p=g_{i}, B d r y=\psi\right)$. Then

$$
\mathcal{L}^{n}\left(\Lambda\left(v_{2}\right) \Delta \Lambda\left(v_{1}\right)\right)=\mathcal{L}^{n}\left(\Omega\left(v_{2}\right) \Delta \Omega\left(v_{1}\right)\right) \leq C(n, \mu)\left\|g_{2}-g_{1}\right\|_{L^{n}} .
$$

4.2 Remark. Note that we do not make any smoothness assumptions about the free boundaries involved.

Proof. Set

$$
\begin{aligned}
& w_{1}:=O P\left(\mathrm{Lap}=\min \left\{g_{1}, g_{2}\right\}, \text { Bdry }=\psi\right) \text { and } \\
& w_{2}:=O P\left(\mathrm{Lap}=\max \left\{g_{1}, g_{2}\right\}, \text { Bdry }=\psi\right) .
\end{aligned}
$$

Since

$$
\mathcal{L}^{n}\left(\Lambda\left(v_{2}\right) \Delta \Lambda\left(v_{1}\right)\right) \subset \mathcal{L}^{n}\left(\Lambda\left(w_{2}\right) \Delta \Lambda\left(w_{1}\right)\right),
$$

it suffices to prove the theorem holds for the $w_{i}$. To simplify the presentation slightly, we will assume that $g_{i} \equiv c_{i}, c_{2}>c_{1}$, and we recall again our auxiliary function

$$
\Phi(x):=\frac{1-\|x\|^{2}}{2 n} .
$$

In the general case, we would replace $\left(c_{2}-c_{1}\right) \Phi$ with the solution to the Dirichlet problem:

$$
\Theta \equiv 0 \text { on } \partial B_{1} \text {, and } \Delta \Theta=-\left|g_{2}-g_{1}\right| \text { in } B_{1} \text {, }
$$

but the argument would otherwise be unchanged. By our comparison principles (see Theorem (2.7)) and other elementary estimates we know the following:

- $w_{2} \leq w_{1} \leq w_{2}+\left(c_{2}-c_{1}\right) \Phi$.

- $\Omega\left(w_{2}\right) \subset \Omega\left(w_{1}\right)$.

- $\Delta w_{1}(x)=c_{1} \chi_{\Omega\left(w_{1}\right)}(x)$, and $\Delta w_{2}(x)=c_{2} \chi_{\Omega\left(w_{2}\right)}(x)$.

Now we define

$$
u(x):=w_{2}(x)+\left(c_{2}-c_{1}\right) \Phi(x)-w_{1}(x) \geq 0,
$$

and note that

$\Delta u \equiv 0$ in $\Omega\left(w_{2}\right), u \equiv 0$ on $\partial B_{1}$, and $u(x) \leq\left(c_{2}-c_{1}\right) \Phi(x)$ on $\partial \Omega\left(w_{2}\right)$.

In this next computation, $\partial B_{1}$ will be short for $\partial B_{1} \cap \partial \Omega\left(w_{1}\right)=\partial B_{1} \cap \partial \Omega\left(w_{2}\right)$, and we make the following definitions: $\Omega_{i}:=\Omega\left(w_{i}\right), F B_{i}:=\partial \Omega_{i}-\partial B_{1}$, and $L:=\Omega_{1}-\Omega_{2}$. 


$$
\begin{aligned}
0 & =\int_{\Omega_{2}} \Delta u \\
& =\int_{\partial \Omega_{2}} \frac{\partial u}{\partial \nu} \\
& =\int_{\partial B_{1}} \frac{\partial u}{\partial \nu}+\int_{F B_{2}} \frac{\partial u}{\partial \nu} \\
& =\int_{\partial B_{1}} \frac{\partial u}{\partial \nu}+\left(c_{2}-c_{1}\right) \int_{F B_{2}} \frac{\partial \Phi}{\partial \nu}-\int_{F B_{2}} \frac{\partial w_{1}}{\partial \nu}
\end{aligned}
$$

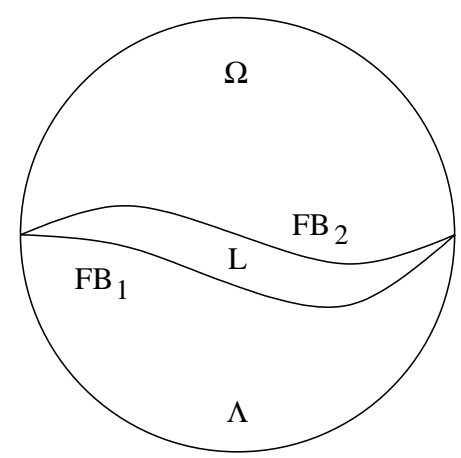

So, since $\int_{F B_{2}} \frac{\partial \Phi}{\partial \nu}=-\int_{\partial B_{1}} \frac{\partial \Phi}{\partial \nu}+\int_{\Omega_{2}} \Delta \Phi$, we easily obtain

$$
\left|\int_{F B_{2}} \frac{\partial w_{1}}{\partial \nu}\right| \leq C\left(c_{2}-c_{1}\right)+\left|\int_{\partial B_{1}} \frac{\partial u}{\partial \nu}\right| .
$$

Since $u \geq 0$ is harmonic, and since for any $x \in \partial B_{1}$ there is an affine function equal to zero at $x$, greater than zero everywhere else on $\partial B_{1}$, and greater than $\Phi$ on $F B_{2}$, we can conclude that on $\partial B_{1}$ we have

$$
\left|\frac{\partial u}{\partial \nu}\right| \leq C\left(c_{2}-c_{1}\right)
$$

and therefore

$$
\left|\int_{F B_{2}} \frac{\partial w_{1}}{\partial \nu}\right| \leq C\left(c_{2}-c_{1}\right)
$$

A hyperplane greater than or equal to $\Phi$, which is equal to $\Phi$ at one point on the boundary.

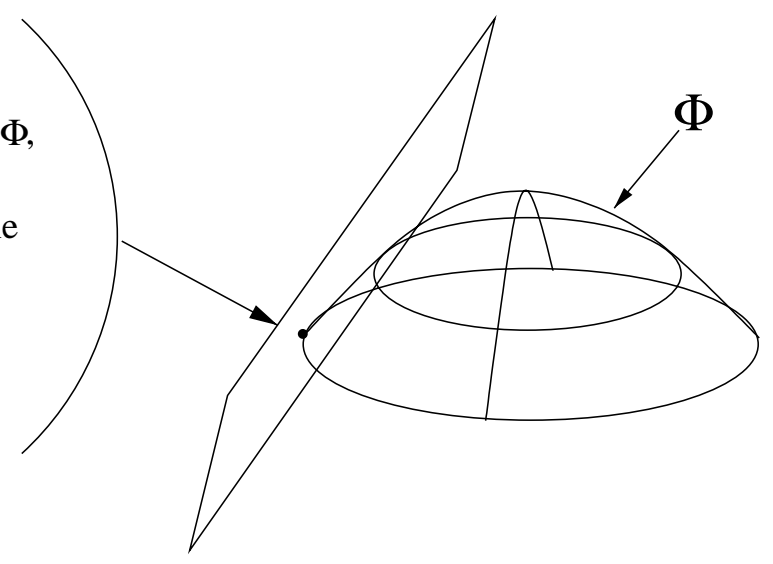


On the other hand

$$
\begin{aligned}
c_{1}|L| & =\int_{L} c_{1} \\
& =\int_{L} \Delta w_{1} \\
& =\int_{F B_{2}} \frac{\partial w_{1}}{\partial \nu} .
\end{aligned}
$$

So

$$
|L| \leq C\left(\frac{c_{2}-c_{1}}{c_{1}}\right)
$$

Q.E.D.

We get immediate consequences for obstacle problems where the Laplacian is only continuous.

4.3 Theorem (Caffarelli's Alternative in Measure Part I). Assuming $w \in \mathcal{P}\left(\lambda, \mu, C\left(B_{1}\right)\right)$ and

$$
\liminf _{r \rightarrow 0} \frac{\left|\Lambda(w) \cap B_{r}\right|}{\left|B_{r}\right|}>0,
$$

then we have

$$
\lim _{r \rightarrow 0} \frac{\left|\Lambda(w) \cap B_{r}\right|}{\left|B_{r}\right|}=\frac{1}{2} .
$$

Proof. We take a sequence $\left\{s_{k}\right\}$ as in Theorem $(2.10)$, so that $w_{s_{k}} \rightarrow \tilde{w}$. By the last corollary we know that (on $B_{1}$ ) we have $\left|\Lambda\left(w_{s_{k}}\right) \Delta \Lambda(\tilde{w})\right| \rightarrow 0$. So for any $r>0$,

$$
\lim _{k \rightarrow \infty}\left(\frac{\left|\Lambda\left(w_{s_{k}}\right) \cap B_{r}\right|}{\left|B_{r}\right|}-\frac{\left|\Lambda(\tilde{w}) \cap B_{r}\right|}{\left|B_{r}\right|}\right)=0 .
$$

But by our rescaling, this equation becomes

$$
\lim _{k \rightarrow \infty}\left(\frac{\left|\Lambda(w) \cap B_{\left(r s_{k}\right)}\right|}{\left|B_{\left(r s_{k}\right)}\right|}-\frac{\left|\Lambda(\tilde{w}) \cap B_{r}\right|}{\left|B_{r}\right|}\right)=0,
$$

which, along with equation (4.8) implies that

$$
\liminf _{r \rightarrow 0} \frac{\left|\Lambda(\tilde{w}) \cap B_{r}\right|}{\left|B_{r}\right|}>0
$$

and therefore

$$
\liminf _{r \rightarrow 0} \frac{m \cdot d \cdot\left(\Lambda(\tilde{w}) \cap B_{r}\right)}{r}>0 .
$$


Now by Theorem $(3.4), F B(\tilde{w})$ is $C^{1, \alpha}$ near zero, and therefore

$$
\lim _{r \rightarrow 0} \frac{\left|\Lambda(\tilde{w}) \cap B_{r}\right|}{\left|B_{r}\right|}=\frac{1}{2} .
$$

Combining this equation with equation (4.11) yields the desired result.

Q.E.D.

Now we introduce another approximating function which will be useful in extending the notion of a regular point to solutions of the obstacle problem where we can only say that the Laplacian has small oscillation.

4.4 Lemma. For any $w \in \mathcal{P}\left(\lambda, \mu, L^{\infty}\right)$, there exists a constant $c_{w} \in[\lambda, \mu]$, such that if $v:=O P\left(\operatorname{Lap}=c_{w}, B d r y=\left.w\right|_{\partial B_{1}}\right)$, then $v \in \mathcal{P}\left(c_{w}\right) .\left(S_{o} 0 \in F B(v)\right.$.) Furthermore, the following estimates are satisfied:

$$
\begin{gathered}
\text { a. } \\
\text { b. } \quad \mathcal{L}^{n}(\Lambda(w) \Delta \Lambda(v))=\mathcal{L}^{n}(\Omega(w) \Delta \Omega(v)) \leq C(n, \mu)(\mu-\lambda) . \\
\text { c. } \quad\|w-v\|_{W^{2, p}\left(B_{1 / 2}\right)} \leq C(n, p, \mu)(\mu-\lambda)^{\frac{1}{p}}, \text { for } p \in(1, \infty) .
\end{gathered}
$$

Proof. First, for any $s \in[\lambda, \mu]$, let ${ }_{s} v:=O P\left(\operatorname{Lap}=s, \operatorname{Bdry}=\left.w\right|_{\partial B_{1}}\right)$. Then $\Lambda\left({ }_{\lambda} v\right) \subset \Lambda(w) \subset \Lambda\left({ }_{\mu} v\right)$, and if $\lambda \leq r<s \leq \mu$, then $\Lambda\left({ }_{r} v\right) \subset \Lambda\left({ }_{s} v\right)$. Now we take a sequence $\left\{s_{j}\right\} \subset[\lambda, \mu]$ such that

$$
\partial \Lambda\left({ }_{s_{j}} v\right) \cap B_{2^{-j}}(0) \neq \phi .
$$

Such a sequence has to exist, since otherwise we would have a ball, $B_{r}(0) \subset$ $\Lambda\left({ }_{r_{0}} v\right) \cap \Omega\left({ }_{r_{1}} v\right)$, with $\left|r_{0}-r_{1}\right|$ arbitrarily small, thereby contradicting measure stability. Now let

$$
c_{w}:=\lim _{j \rightarrow \infty} s_{j} .
$$

Clearly $v(0):={ }_{w} v(0)=0$, so $0 \in \Lambda(v)$. On the other hand, our nondegeneracy statement, Theorem (2.1), shows that there are points of $\Omega(v)$ which are arbitrarily close to 0 , so $0 \in F B(v)$.

Now estimates (a) and (b) follow immediately from the application of Theorems (4.1) and (2.7), so it remains to prove (c). We start by defining $u:=w-v \cdot u$ is 0 on $\partial B_{1}$, and

$$
\Delta u=\Delta w \chi_{\Omega(w)}-c_{w} \chi_{\Omega(v)} .
$$


So, by (b) we know that $|\Delta u| \leq C(\mu-\lambda)$, except on a set whose measure is less than $C(\mu-\lambda)$. Thus,

$$
\|\Delta u\|_{L^{p}\left(B_{1 / 2}\right)} \leq C(\mu-\lambda)+\mu\left(\int_{\Omega(w) \Delta \Omega(v)}|1|^{p}\right)^{\frac{1}{p}} \leq C(\mu-\lambda)^{\frac{1}{p}} .
$$

Calderón-Zygmund Estimates now yield the desired result. Q.E.D.

Now we classify the points of a free boundary where the Laplacian of the obstacle does not have to be continuous, but merely satisfies a small oscillation condition.

For the rest of this section we make the following assumptions:

1. $\mu$ is a fixed positive number.

2. $\epsilon$ is a fixed positive number less than $1 / 4$.

In the next theorem, all constants will depend on $n, \mu$, and $\epsilon$, unless otherwise stated.

4.5 Theorem (Caffarelli's Alternative in Measure Part II). There exists a $\lambda_{0} \in(0, \mu)$ such that if $\lambda_{0} \leq \lambda \leq \mu$, then for any $w \in \mathcal{P}\left(\lambda, \mu, L^{\infty}\right)$ we have either

a. 0 is a Singular Point of $F B(w)$ :

$$
\limsup _{r \rightarrow 0} \frac{\left|\Lambda(w) \cap B_{r}\right|}{\left|B_{r}\right|} \leq \epsilon
$$

or

b. 0 is a Regular Point of $F B(w)$ :

$$
\liminf _{r \rightarrow 0} \frac{\left|\Lambda(w) \cap B_{r}\right|}{\left|B_{r}\right|} \geq \frac{1}{2}-\epsilon .
$$

In fact there exists an $r_{0} \in(0,1)$ and $a \tau \in(0,1)$ such that if

$$
\frac{\left|\Lambda(w) \cap B_{t}\right|}{\left|B_{t}\right|}>\epsilon, \text { for some } t \leq r_{0}
$$

then for all $r \leq \tau t$ we have

$$
\frac{\left|\Lambda(w) \cap B_{r}\right|}{\left|B_{r}\right|} \geq \frac{1}{2}-\epsilon
$$


4.6 Remark. Since our rescaling does not effect the $L^{\infty}$ bounds on the Laplacian of our obstacle from above or below, this result classifies all points of $F B(w)$ as being regular or singular as defined above.

Proof. The second part of the theorem is stronger than the first part, and we will go straight to its proof. By rescaling back and forth, we can assume without loss of generality that $t=r_{0}$. (i.e. (4.14) holds with $t=r_{0}$.) If we take $\mu-\lambda_{0}$ sufficiently small, then the function $v$, given by Lemma (4.4), satisfies

$$
\frac{\left|\Lambda(v) \cap B_{r_{0}}\right|}{\left|B_{r_{0}}\right|} \geq \frac{\epsilon}{2},
$$

and therefore

$$
\text { m.d. }\left(\Lambda(v) \cap B_{r_{0}}\right) \geq C(n) r_{0} \epsilon .
$$

Now if $r_{0}$ is small enough, then by Theorem (3.4) we conclude that $\partial \Lambda(v)$ is $C^{1, \alpha}$ in an $r_{0}^{2}$ neighborhood of the origin. Furthermore, if $F B(v)=\left\{\left(x^{\prime}, x_{n}\right) \mid x_{n}=\right.$ $\left.f\left(x^{\prime}\right)\right\}$, then we have the following bound (in $B_{r_{0}^{2}}$ ):

$$
\|f\|_{C^{1, \alpha}} \leq \frac{C(n)}{r_{0}} .
$$

But, because of this bound, there exists a $\gamma<1$ such that if $\rho_{0}:=\gamma r_{0}<r_{0}$, then

$$
\frac{\left|\Lambda(v) \cap B_{\rho_{0}}\right|}{\left|B_{\rho_{0}}\right|}>\frac{1-\epsilon}{2} .
$$

Now by once again requiring $\mu-\lambda_{0}$ to be sufficiently small, we get

$$
\frac{\left|\Lambda(w) \cap B_{\rho_{0}}\right|}{\left|B_{\rho_{0}}\right|}>\frac{1}{2}-\epsilon .
$$

Finally, since $\frac{1}{2}-\epsilon$ is strictly greater than $\epsilon$, we can rescale $B_{\rho_{0}}$ to a ball with a radius close to $r_{0}$, and then repeat. Since we have a little margin for error in our rescaling, after we repeat this process enough times we will have a small enough radius (which we call $\tau r_{0}$ ), to ensure that for all $r \leq \tau r_{0}$ we have

$$
\frac{\left|\Lambda(w) \cap B_{r}\right|}{\left|B_{r}\right|}>\frac{1}{2}-\epsilon .
$$

Q.E.D.

4.7 Remark. Our regular points are also regular in the sense of the Wiener criterion (see $[\mathrm{GT}]$ ). 
4.8 Corollary (The Set of Regular Points Is Open). If we take $w$ and $\lambda$ as in the previous theorem, then the set of regular points of $F B(w)$ is an open subset of $F B(w)$.

Proof. Take $r_{0}$ and $\tau$ as in the last theorem. By changing coordinates and rescaling, we can assume that 0 is a regular point of $F B(w)$. Since 0 is regular, there exists an $s \leq r_{0}$ such that

$$
\frac{\left|\Lambda(w) \cap B_{s}\right|}{\left|B_{s}\right|}>\epsilon
$$

Now by the previous theorem we know that if $r \leq \tau s$, then

$$
\frac{\left|\Lambda(w) \cap B_{r}\right|}{\left|B_{r}\right|} \geq \frac{1}{2}-\epsilon
$$

Again, since $\frac{1}{2}-\epsilon>\epsilon$, we have some margin for error. If $\gamma:=\left\|x_{0}\right\|$ is sufficiently small, and $x_{0} \in F B(w)$, then

$$
\frac{\left|\Lambda(w) \cap B_{(\tau s-\gamma)}\left(x_{0}\right)\right|}{\left|B_{(\tau s-\gamma)}\left(x_{0}\right)\right|}>\epsilon,
$$

and therefore for any $r \leq \tau(\tau s-\gamma)$ we have

$$
\frac{\left|\Lambda(w) \cap B_{r}\left(x_{0}\right)\right|}{\left|B_{r}\left(x_{0}\right)\right|} \geq \frac{1}{2}-\epsilon .
$$

Thus, $x_{0}$ is a regular point.

Q.E.D.

4.9 Remark. Combining this theorem with Theorem (4.3) allows us to conclude that the regular points of the free boundary also form an open set when the Laplacian of the obstacle is continuous.

\section{The Uniform Stability at Regular Points}

Now we prove a result which gives uniform stability of the free boundary near regular points. Previously, the way to get uniform stability near a free boundary point was to use nondegeneracy to get one direction, and use the monotonicity of the "normal" derivative to get the other direction. (See for example the proof of Theorem (2.10)d.) Again we improve the theory from [C3] by eliminating the square root from our estimate, at least in the case where our solution is sufficiently close to $\left(x_{n}^{+}\right)^{2}$. This improvement will be essential to prove optimal regularity of the free boundary. Finally, we will show that linear stability is always optimal for smooth free boundaries by proving a linear nondegeneracy result.

We start with a slight improvement on one of our comparison principles. 
5.1 Lemma (Comparison Principle). Let $w:=O P(\operatorname{Lap}=1, B d r y=\psi)$, $w_{\epsilon}:=O P($ Lap $=1+\epsilon, B d r y=\psi)$, and let $v$ denote the solution of the Dirichlet problem on $\Omega(w)$ with zero boundary data, and $\Delta v \equiv 1$ in $\Omega(w)$. Then in $\Omega(w)$ we have:

$$
w+\epsilon v \leq w_{\epsilon} \leq w
$$

Proof. Let $\Theta:=w+\epsilon v-w_{\epsilon}$, and assume that $\Theta$ has a positive maximum at $x_{0}$. Then $w\left(x_{0}\right)>0$, so $\Delta \Theta\left(x_{0}\right)=1+\epsilon-\Delta w_{\epsilon}\left(x_{0}\right) \geq 0$, and therefore $x_{0}$ would have to be on $\partial \Omega(w)$. But $\Theta \equiv 0$ on $\partial \Omega(w)$, which gives us our contradiction. Q.E.D.

5.2 Remark. No matter what $\psi$ is, we still know that

$$
0 \geq v(x) \geq-\Phi(x)=\frac{\|x\|^{2}-1}{2 n} \geq-\frac{1}{2 n} .
$$

So assuming that $F B(w)$ is smooth, we will have $\|\nabla v\|$ bounded by a constant $\tilde{C}$ on $B_{1 / 2} \cap \Omega(w)$, depending on only the smoothness of $F B(w) \cap B_{5 / 8}$. (We will be able to assume uniform $C^{1, \alpha}$ smoothness of $F B(w) \cap B_{5 / 8}$ and then either invoke Kinderlehrer and Nirenberg's higher regularity results to get uniform $C^{2, \alpha}$ smoothness (see Theorem (3.8) ) so that we can invoke Schauder's estimates, or use results of Gilbarg and Hörmander (see [GH]) which extend Schauder's estimates to the $C^{1, \alpha}$ case.) Since $v$ is zero on $F B(w)$ we can conclude that for any $x \in B_{1 / 2} \cap \Omega(w)$ we have

$$
|v(x)| \leq \tilde{C} d(x, F B(w)) .
$$

So now we want some stability of the smoothness of the free boundary under perturbations of the Laplacian.

5.3 Lemma (Persistent Smoothness). There exist constants $\delta_{0}$ and $\epsilon_{0}$, which depend only on $n$, such that if

$$
\left|\psi(x)-\frac{\left(x_{n}^{+}\right)^{2}}{2}\right| \leq \delta_{0},
$$

and

$$
w=O P(\operatorname{Lap}=c, B d r y=\psi),
$$

where $|1-c| \leq \epsilon_{0}$, then $F B(w) \cap B_{3 / 4}=\left\{x_{n}=f\left(x^{\prime}\right)\right\}$ where $f$ is universally bounded in $C^{1, \alpha}$. (i.e., the smoothness is independent of $w$.)

Proof. We start by considering the case where $c=1$, and we will let $w_{c}:=$ $O P(\operatorname{Lap}=c$, Bdry $=\psi)$. By our first comparison principle (Theorem (2.7) a) 
and by our basic interpolation estimate (Theorem (2.6)), we know that on $B_{7 / 8}$ we have

$$
\left\|w_{1}(x)-\frac{\left(x_{n}^{+}\right)^{2}}{2}\right\|_{\infty} \leq \delta_{0}, \text { and }\left\|\nabla w_{1}(x)-\left(x_{n}^{+}\right) e_{n}\right\|_{\infty} \leq \tilde{\sigma}\left(\delta_{0}\right),
$$

where $\tilde{\sigma}$ is a universal modulus of continuity. So nondegeneracy implies

$$
F B\left(w_{1}\right) \subset\left\{x_{n} \geq-\sigma_{1}\left(\delta_{0}\right)\right\},
$$

and the last estimate on the gradient implies

$$
F B\left(w_{1}\right) \subset\left\{x_{n} \leq \sigma_{2}\left(\delta_{0}\right)\right\}
$$

where the $\sigma_{i}$ are universal moduli of continuity. Thus, by shrinking $\delta_{0}$ we can make $F B\left(w_{1}\right) \cap B_{7 / 8}$ uniformly close to $\left\{x_{n}=0\right\}$. Now Theorem (3.4) gives us the desired regularity since we can ensure that for any $x_{0} \in F B\left(w_{1}\right) \cap B_{7 / 8}$ we have a small $\rho_{0} \gg\left[\sigma_{1}\left(\delta_{0}\right)+\sigma_{2}\left(\delta_{0}\right)\right]$ such that m.d. $\left(\Lambda \cap B_{\rho_{0}}\left(x_{0}\right)\right) \geq \rho_{0} / 4$.

The shaded region is the portion of the ball which MUST lie in the zero set. So its minimum diameter is a "worst case."

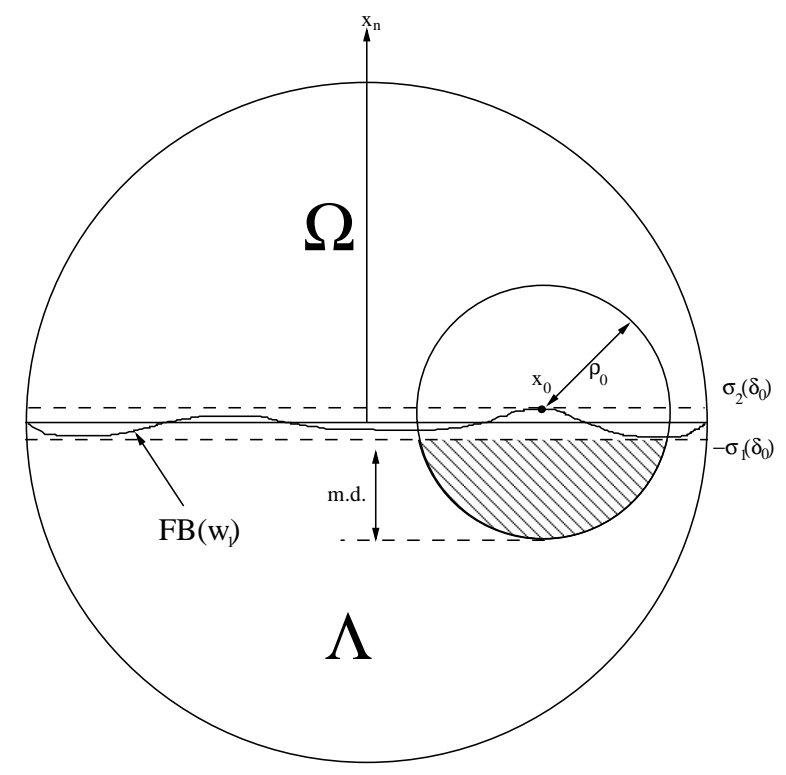

Now we need to deal with the case $c \neq 1$. We use our third comparison principle (Theorem $(2.7) \mathrm{c}$ ) to get on $B_{3 / 4}$ that

$$
\begin{aligned}
& \left\|w_{c}(x)-w_{1}(x)\right\|_{\infty} \leq \epsilon_{0}, \text { and } \\
& \left\|\nabla w_{c}(x)-\nabla w_{1}(x)\right\|_{\infty} \leq \tilde{\sigma}\left(\epsilon_{0}\right),
\end{aligned}
$$

so that the triangle inequality gives

$$
\left\|w_{c}(x)-\frac{\left(x_{n}^{+}\right)^{2}}{2}\right\|_{\infty} \leq \delta_{0}+\epsilon_{0}
$$


and

$$
\left\|\nabla w_{c}(x)-\left(x_{n}^{+}\right) e_{n}\right\|_{\infty} \leq \tilde{\sigma}\left(\delta_{0}\right)+\tilde{\sigma}\left(\epsilon_{0}\right) .
$$

The argument is now identical to the one for the first case.

Q.E.D.

We can now prove the main result of this section.

5.4 Theorem (Linear Stability of the Free Boundary). Suppose that

$$
\left|\psi(x)-\frac{\left(x_{n}^{+}\right)^{2}}{2}\right| \leq \delta_{0},
$$

where $\delta_{0}$ is taken as in the previous lemma, and let $w:=O P(\operatorname{Lap}=1, B d r y=\psi)$ and $w_{\epsilon}:=O P(\operatorname{Lap}=1+\epsilon, B d r y=\psi)$.

Then if $|\epsilon| \leq \epsilon_{0}$ (with $\epsilon_{0}$ as in the previous lemma), then on $B_{1 / 2}$ we have

$$
\operatorname{dist}\left(F B(w), F B\left(w_{\epsilon}\right)\right) \leq C|\epsilon|
$$

Proof. For simplicity, we will assume that $0<\epsilon$. The case where $0>\epsilon \geq-\epsilon_{0}$ can be treated similarly. We satisfy all of the conditions of Lemmas (5.1) and (5.3), and, with $v$ defined as in Lemma (5.1), we also satisfy equation (5.3). Putting these equations together, we get

$$
w(x)-\epsilon \tilde{C} d(x, F B(w)) \leq w_{\epsilon}(x) \leq w(x) .
$$

On the other hand, by Theorem (3.3) c, we know that $w(x) \geq C d(x, F B(w))^{2}$. So,

$$
C d(x, F B(w))^{2}-\epsilon \tilde{C} d(x, F B(w)) \leq w_{\epsilon}(x) \leq w(x),
$$

which forces $w_{\epsilon}(x)>0$ once $d(x, F B(w))>C \epsilon$.

Q.E.D.

5.5 Remark. Everything in this section so far has a nonlinear analogue. In particular, Lemma (5.1) can be proven by taking the test function $v$ to be a solution of the following boundary value problem

$$
\begin{aligned}
\mathcal{M}^{-}\left(D^{2} u, \lambda, 2 n \Lambda\right) & =1 & & \text { in } \Omega(w) \\
u & =0 & & \text { on } \partial \Omega(w) .
\end{aligned}
$$

(See $[\mathrm{CC}]$ for the definition of Pucci's extremal operator $\mathcal{M}^{-}$.) Equation (5.3) still holds for smooth free boundaries as can be verified most easily by constructing simple barriers. Persistent smoothness is proven by first proving a square root uniform stability estimate (i.e. uniform stability the "oldfashioned" way), and then using Ki-ahm Lee's nonlinear Caffarelli Alternative 
to show that the perturbed free boundary is still $C^{1, \alpha}$. At that point the proof of the linear control of the free boundary is identical to the one above. The last thing we will note in this section is the linear nondegeneracy of smooth free boundaries under a perturbation.

The same methods as in the proof of lemma (5.3) can also be used to prove the following lemma.

5.6 Lemma (General Persistent Smoothness). Suppose $w \in \widehat{\mathcal{P}}(1)$ and $F B(w) \cap B_{3 / 4}=\left\{x_{n}=f\left(x^{\prime}\right)\right\}$ where

$$
\|f\|_{C^{1, \alpha}\left(\overline{B_{3 / 4}^{\prime}}\right)} \leq \gamma<+\infty .
$$

If $\gamma$ is sufficiently small (depending only on $n$ and $\alpha$ ), then there exists an $\epsilon_{0}=$ $\epsilon_{0}(n, \gamma, \alpha)>0$ such that if $\|1-c\| \leq \epsilon_{0}$, and $w_{c}:=O P\left(\operatorname{Lap}=c, B d r y=\left.w\right|_{\partial B_{1}}\right)$ then $F B\left(w_{c}\right) \cap B_{5 / 8}=\left\{x_{n}=f\left(x^{\prime}, c\right)\right\}$ with

$$
\|f(\cdot, c)\|_{C^{1, \alpha}\left(\overline{B_{5 / 8}^{\prime}}\right)} \leq 2 \gamma<+\infty \text {. }
$$

5.7 Theorem (Linear Nondegeneracy). Take $\epsilon_{0}$ and $\gamma$ as in the previous lemma. Suppose $w \in \widehat{\mathcal{P}}(1)$ and $\Omega(w) \cap B_{3 / 4}=\left\{x_{n}=f\left(x^{\prime}\right)\right\}$ with

$$
\|f\|_{C^{1, \alpha}\left(\overline{B_{3 / 4}^{\prime}}\right)} \leq \gamma
$$

Then there exist constants $\beta$ and $\alpha_{0}$ such that if $|\epsilon| \leq \min \left\{\alpha_{0}, \epsilon_{0}\right\}$, and $w_{\epsilon}:=$ $O P\left(\operatorname{Lap}=1+\epsilon, B d r y=\left.w\right|_{\partial B_{1}}\right)$ then $\left\{F B(w)+B_{\beta|\epsilon|}\right\} \cap F B\left(w_{\epsilon}\right)=\phi$.

Proof. Again for simplicity, we will assume that $0<\epsilon \leq \epsilon_{0}$. The case where $0>\epsilon \geq-\epsilon_{0}$ can be treated similarly. Let $\tilde{v}$ be the solution of

$$
\begin{array}{rll}
\Delta \tilde{v}=1 & \text { in } & \Omega\left(w_{\epsilon}\right) \\
\tilde{v}=0 & \text { on } & \partial \Omega\left(w_{\epsilon}\right) .
\end{array}
$$

Now observe that the function $h(x):=w(x)+\epsilon \tilde{v}-w_{\epsilon}(x)$ is harmonic in $\Omega\left(w_{\epsilon}\right)$. The maximum principle now implies that $w_{\epsilon}(x)-\epsilon \tilde{v}(x)<w(x)$. Now by the theory due to Kinderlehrer and Nirenberg we can conclude that $F B(w)$ and $F B\left(w_{\epsilon}\right)$ are $C^{\infty}$ in $B_{5 / 8}$, and furthermore the $C^{2, \alpha}$ norms of these free boundaries are uniformly bounded in $B_{5 / 8}$ by compactness. (See Theorem (3.8) in this paper or see $[\mathrm{KN}]$ for all of the details.) Now by combining the Schauder Theory with the Hopf Lemma (see Lemma 3.4 of [GT]) we can deduce the existence of a constant $\alpha_{0}$, such that if $\operatorname{dist}\left(x, B_{1 / 2} \cap \Lambda\left(w_{\epsilon}\right) \leq \alpha_{0}\right.$, then $\tilde{v}(x) \leq-\gamma \operatorname{dist}\left(x, \Lambda\left(w_{\epsilon}\right)\right.$. We can combine the estimates above and our $C^{1,1}$ regularity of $w$ to get for any $x$ within $\alpha_{0}$ of $B_{1 / 2} \cap \Lambda\left(w_{\epsilon}\right)$

$$
\epsilon \gamma d\left(x, \Lambda\left(w_{\epsilon}\right)\right) \leq-\epsilon \tilde{v}(x) \leq w_{\epsilon}(x)-\epsilon \tilde{v}(x)<w(x) \leq C(n) d(x, \Lambda(w))^{2} .
$$


Let $\beta:=\min \left\{\alpha_{0}, \gamma / 8 C(n)\right\}$ with the same $C(n)$ as in equation (5.13) . Now suppose $x_{1} \in F B(w) \cap B_{1 / 2}, x_{2} \in F B\left(w_{\epsilon}\right)$, and $\operatorname{dist}\left(x_{1}, x_{2}\right) \leq \beta \epsilon$. Choose $x_{0} \in \Omega\left(w_{\epsilon}\right)$ such that $\operatorname{dist}\left(x_{0}, \Lambda\left(w_{\epsilon}\right)\right)=\operatorname{dist}\left(x_{0}, x_{2}\right)=\beta \epsilon$. So we now have

$$
\begin{aligned}
\beta \gamma \epsilon^{2} & =\epsilon \gamma d\left(x_{0}, \Lambda\left(w_{\epsilon}\right)\right) \\
& <C(n) d(x, \Lambda(w))^{2} \\
& \leq C(n)\left[d\left(x_{0}, x_{2}\right)+d\left(x_{2}, x_{1}\right)\right]^{2} \\
& \leq C(n)[2 \beta \epsilon]^{2} \\
& =4 C(n) \beta^{2} \epsilon^{2}
\end{aligned}
$$

which contradicts our definition of $\beta$.

Q.E.D.

5.8 Remark. Although we have assumed that $F B(w)$ in addition to its smoothness is also the graph of a function on $B_{3 / 4}$, this assumption is not crucial but only convenient. By scaling and covering lemmas, we can get around this requirement, as all we really need is to be able to use the Hopf Lemma as above.

Before we can proceed, we need a better understanding of flatness.

\section{When Flatness Implies Smoothness}

6.1 Definition (Reifenberg Flatness). Let $S \subset \mathbb{R}^{n}$ be a locally compact set, and let $\delta>0$. Then $S$ is $\delta$-Reifenberg flat if for each compact $K \subset \mathbb{R}^{n}$, there exists a constant $R_{K}>0$ such that for every $x \in K \cap S$ and every $r \in\left(0, R_{K}\right]$ we have a hyperplane $L(x, r)$ containing $x$ such that

$$
D\left(L(x, r) \cap B_{r}(x), S \cap B_{r}(x)\right) \leq 2 r \delta .
$$

Here $D$ denotes the Hausdorff distance: If $A, B \subset \mathbb{R}^{n}$, then

$$
D(A, B):=\max \left\{\sup _{a \in A} d(a, B), \sup _{b \in B} d(b, A)\right\} .
$$

Note that unless $\delta$ is small, the last definition will be satisfied by any set, so we will assume that $\delta \in\left(0, \frac{1}{10}\right)$ henceforward. We will say that a set is Reifenberg flat if it is $\delta$-Reifenberg flat for some $\delta \in\left(0, \frac{1}{10}\right)$. We also define the following quantity, which we call the modulus of flatness, to get a more quantitative and uniform measure of flatness:

$$
\theta_{K}(r):=\sup _{0<\rho \leq r}\left(\sup _{x \in S \cap K} \frac{D\left(L(x, \rho) \cap B_{\rho}(x), S \cap B_{\rho}(x)\right)}{\rho}\right) .
$$


Finally, we will say that $S$ is a Reifenberg vanishing set, if for any compact $K \subset S$

$$
\lim _{r \rightarrow 0} \theta_{K}(r)=0 .
$$

Reifenberg flatness was introduced by Reifenberg in $[R]$, and is studied in more detail by Toro and Kenig in several papers. In particular, the following theorem is known (see [T1]).

6.2 Theorem (Reifenberg,Toro). There exists a $\delta=\delta(n)$ such that if $S$ is $\delta$-Reifenberg flat then $S$ is locally homeomorphic to a disc. Furthermore, this homeomorphism yields a locally Hölder parametrization for $S$. Thus, there exists a $\beta=\beta(\delta) \in(0,1)$ such that $S$ is a $C^{0, \beta}(n-1)$-dimensional submanifold. Furthermore, $\beta \rightarrow 1$ as $\delta \rightarrow 0$.

6.3 Remark. If a set is Reifenberg flat, then we can get estimates of its minimum diameter on different scales. We simply use $L(x, r)$ shifted by the Reifenberg flatness in one direction and then in the other. (We will refer to these planes as the bounding planes.) On the other hand, we can have very degenerate sets with small minimum diameter at all scales which are not Reifenberg flat. (e.g., A single point.)

With these definitions, we can restate some of the theorems about regular points. Again we assume $\mu$ and $\epsilon$ are fixed as before, we take $\lambda$ and $w$ as in Theorem (4.5), and we start with $\lambda_{0}$ also as in Theorem (4.5), but we allow it to approach $\mu$ a little if necessary (i.e., $\lambda_{0}=\lambda_{0}(n, \epsilon, \mu)$ as before, but $\mu-\lambda_{0}$ might be smaller than in Theorem (4.5) ).

6.4 Theorem (Flatness of Regular Points). There exists an $r_{0} \in(0,1)$ and $a \tau \in(0,1)$ such that if

$$
\frac{\left|\Lambda(w) \cap B_{t}\right|}{\left|B_{t}\right|}>\epsilon, \text { for some } t \leq r_{0},
$$

then $\Lambda(w) \cap \overline{B_{\tau t}}$ is a $\delta$-Reifenberg flat set, for some $\delta=\delta\left(n, \mu, \lambda_{0}, \epsilon\right)$.

In fact, if $w \in \widehat{\mathcal{P}}\left(\lambda, \mu, C\left(\bar{B}_{1}\right)\right)$, then $F B(w)$ is a Reifenberg vanishing set near the origin.

Proof. The proof of this theorem is almost the same as the proof of Theorem (4.5). We simply have to observe that equation (4.18) followed from a stronger statement about the smoothness of $F B(v)$. (See the proof of Theorem (4.5) for the precise definition of $v$, which is an approximation to $w$ with $0 \in F B(v)$.) Now the flatness of $F B(w)$ follows from the uniform stability near regular points and the flatness of $F B(v)$. Q.E.D. 
Now we relate Reifenberg flatness to more classical notions of smoothness. But first we mention that there are examples of Reifenberg vanishing sets which do not admit tangent planes. There are examples in the little-o Zygmund class (see [Z] or [T2]), and there will be a simple example in this paper. But for now, we will state a positive result. We start with a trivial lemma.

6.5 Lemma (Alternate Dini Condition). Let $\sigma$ be a modulus of continuity. Then

$$
\int_{0}^{1} \frac{\sigma(r)}{r} d r<\infty
$$

if and only if

$$
\sum_{k=0}^{\infty} \sigma\left(2^{-k}\right)<\infty
$$

Proof. Simply observe that

$$
\begin{aligned}
\frac{1}{2} \sigma\left(2^{-k}\right) & =2^{-k} \frac{\sigma\left(2^{-k}\right)}{2^{-k+1}} \\
& \leq \int_{2^{-k}}^{2^{-k+1}} \frac{\sigma(r)}{r} d r \\
& \leq 2^{-k} \frac{\sigma\left(2^{-k+1}\right)}{2^{-k}}=\sigma\left(2^{-k+1}\right)
\end{aligned}
$$

and sum over $k$.

Q.E.D.

6.6 Remark. In fact we have shown that the quantities in equations (6.6) and (6.7) are comparable (when they are finite), and obviously the proof remains the same if we replace the number " 2 " everywhere with any number more than one.

In the next theorem we will denote the angle between $A$ and $B$ by $\measuredangle(A, B)$, and take $K=S$ so that $\theta(r)$ will be short for $\theta_{S}(r)$.

6.7 Theorem (Condition for Differentiability). Let $S \subset \mathbb{R}^{n}$ be a compact set. If $S$ is a Reifenberg vanishing set with

$$
\int_{0}^{1} \frac{\theta(r)}{r} d r<\infty
$$

then $S$ is locally the graph of a $C^{1}$ function. Furthermore we get the following estimate on the modulus of continuity of the derivative of the local parametrization. There exists a constant $C_{0}$ such that if $\rho>0$ is small enough to imply

$$
\int_{0}^{\rho} \frac{\theta(r)}{r} d r<C_{0}
$$


then for any $z \in S$, there exists a system of coordinates and a function $f$ such that

$$
S \cap B_{\rho}(z)=\left\{\left(x^{\prime}, x_{n}\right) \mid x_{n}=f\left(x^{\prime}\right)\right\}, \quad \frac{\partial f}{\partial x^{\prime}}(z)=0,
$$

and for any $j<n$,

$$
\left\|\frac{\partial f}{\partial x_{j}}\left(x^{\prime}\right)-\frac{\partial f}{\partial x_{j}}\left(y^{\prime}\right)\right\| \leq C_{1} \int_{0}^{\left\|x^{\prime}-y^{\prime}\right\|} \frac{\theta(r)}{r} d r
$$

for $x^{\prime}$ and $y^{\prime}$ in $B_{\rho / 2}^{\prime}\left(z^{\prime}\right)$.

Before we prove this theorem, we first provide some intuition as to why it must be true.

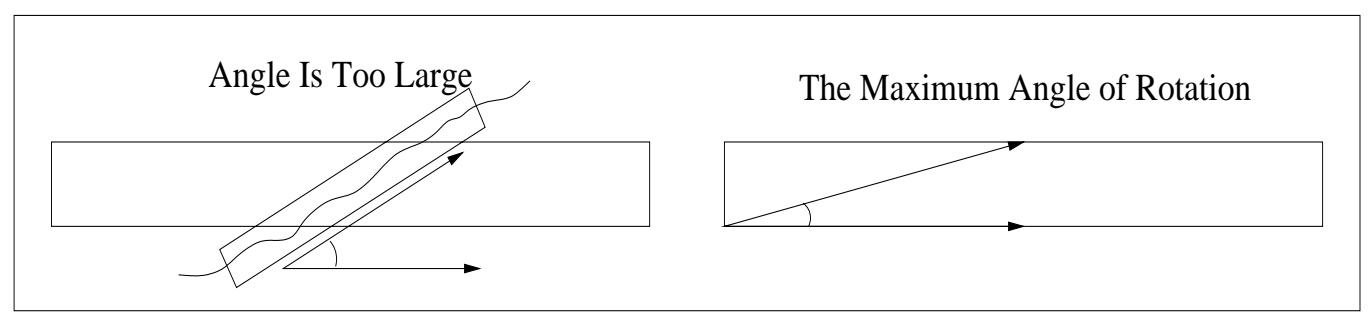

As we look at the bounding hyperplanes of our set on smaller and smaller scales, they can start rotating. On the other hand, the minimum diameter of our set intersected with a ball of radius $r$ gives us a bound for the rotation of the bounding planes at the scale $r / 2$. If the angle of rotation is too large, then the hyperplanes will intersect in such a way as to make it impossible that $S \cap B_{r}$ was contained between the original pair of hyperplanes. So we can bound the angle between the planes at scale $r$ and the planes at scale $r / 2$ by

$$
2 \arctan (\theta(r)) \leq 2 \theta(r) \text { for } \theta(r) \text { sufficiently small. }
$$

Similarly, the next angle of rotation will be bounded by $2 \theta(r / 2)$. So if

$$
\sum_{k=1}^{\infty} \theta\left(2^{-k} r\right)<\infty
$$

then the normal vector to these planes will have to converge. But by our last lemma, equation (6.12) holds by our assumptions on $\theta(r)$.

Proof. The discussion above shows that for any $x \in S$ we can associate a unique unit vector $\vec{v}(s)$ (equal to the limit of the normals of the approximating planes) which we will show is a genuine normal to $S$. It is also clear that $\vec{v}(s)$ is a continuous function of $s$, with a modulus of continuity which we can express 
with the following equation measuring the angle between any two of these vectors which are sufficiently close.

$$
\measuredangle(\vec{v}(s), \vec{v}(t)) \leq 4 \int_{0}^{\|s-t\|} \frac{\theta(r)}{r} d r .
$$

Now by an affine transformation we can assume that $0 \in S$ and $\vec{v}(0)=e_{n}$. We make the following definition:

$$
f\left(x^{\prime}\right):=2\left\|x^{\prime}\right\|\left(\int_{0}^{4\left\|x^{\prime}\right\|} \frac{\theta(r)}{r} d r+\theta\left(4\left\|x^{\prime}\right\|\right)\right)
$$

so

$$
f\left(x^{\prime}\right) \leq o\left(\left\|x^{\prime}\right\|\right)
$$

and we will show that $S \cap B_{\rho} \subset\left\{\left(x^{\prime}, x_{n}\right) \mid-10 f\left(x^{\prime}\right) \leq x_{n} \leq 10 f\left(x^{\prime}\right)\right\}$. At that point Theorem (6.2) combined with the estimates above yield the desired result.

By symmetry, it suffices to prove that $S \cap B_{\rho} \subset\left\{\left(x^{\prime}, x_{n}\right) \mid x_{n} \leq 10 f\left(x^{\prime}\right)\right\}$. Suppose not. Then there exists an $s=\left(s^{\prime}, s_{n}\right) \in S \cap B_{\rho}$ with

$$
s_{n}>10 f\left(s^{\prime}\right)=20\left\|s^{\prime}\right\|\left(\int_{0}^{4\left\|s^{\prime}\right\|} \frac{\theta(r)}{r} d r+\theta\left(4\left\|s^{\prime}\right\|\right)\right) .
$$

So the line $\ell$ through the origin and $s$ makes an angle with the plane $\left\{x_{n}=0\right\}$ of at least

$\arctan \left(20\left[\int_{0}^{4\left\|s^{\prime}\right\|} \frac{\theta(r)}{r} d r+\theta\left(4\left\|s^{\prime}\right\|\right)\right]\right) \geq 20\left(\int_{0}^{4\left\|s^{\prime}\right\|} \frac{\theta(r)}{r} d r+\theta\left(4\left\|s^{\prime}\right\|\right)\right)$.

On the other hand, there is a plane $L(0,\|s\|)$ through the origin with a distance to $s$ which is bounded by

$$
\|s\| \theta(\|s\|) \leq 2\left\|s^{\prime}\right\| \theta\left(2\left\|s^{\prime}\right\|\right)
$$

So the angle between this plane and $\ell$ is less than

$$
2 \theta(\|s\|) \leq 2 \theta\left(2\left\|s^{\prime}\right\|\right) \leq 2 \theta\left(4\left\|s^{\prime}\right\|\right) .
$$

Hence we can conclude

$$
\measuredangle\left(L(0,\|s\|),\left\{x_{n}=0\right\}\right) \geq 18\left(\int_{0}^{4\left\|s^{\prime}\right\|} \frac{\theta(r)}{r} d r+\theta\left(4\left\|s^{\prime}\right\|\right)\right) .
$$


Now we need to start making our way back to the origin. We consider the planes: $L\left(0,\|s\| 2^{-k}\right)$ as $k \rightarrow \infty$. By the way we chose our coordinate system, we know that

$$
\lim _{k \rightarrow \infty} \measuredangle\left(L\left(0,\|s\| 2^{-k}\right),\left\{x_{n}=0\right\}\right)=0 .
$$

On the other hand, we also know that

$$
\measuredangle\left(L\left(0,\|s\| 2^{-k}\right), L\left(0,\|s\| 2^{-k-1}\right)\right) \leq 2 \theta\left(\|s\| 2^{-k}\right)
$$

which implies

$$
\begin{aligned}
\measuredangle\left(L(0,\|s\|), L\left(0,\|s\| 2^{-k}\right)\right) & \leq 2 \sum_{j=0}^{k} \theta\left(\|s\| 2^{-j}\right) \\
& \leq 2 \sum_{j=0}^{\infty} \theta\left(\|s\| 2^{-j}\right) \\
& \leq 4 \int_{0}^{\|s\|} \frac{\theta(r)}{r} d r \\
& \leq 4 \int_{0}^{4\|s\|} \frac{\theta(r)}{r} d r .
\end{aligned}
$$

Combining this last estimate with equations (6.16) and (6.17), yields the desired contradiction.

Finally, now that we know $\vec{v}(s)$ is a genuine normal vector, the estimate (6.11) follows immediately from equation (6.13). Q.E.D.

\section{Sharp Results When the Laplacian of the Obstacle Is Continuous}

7.1. Theorem (The Modulus of Flatness of the Free Boundary Is Bounded by the Modulus of Continuity of the Laplacian). Let $w \in \widehat{\mathcal{P}}\left(\lambda, \mu, C^{\sigma}\left(\bar{B}_{1}\right)\right)$, where $C^{\sigma}\left(\bar{B}_{1}\right)$ is the set of continuous functions with modulus of continuity equal to $\sigma$, and suppose further that $F B(w) \cap \overline{B_{3 / 4}}$ is $\delta$-Reifenberg flat for some $\delta$ less than or equal to some sufficiently small (but universal) $\delta_{0}$. Then the modulus of flatness, $\theta$, of $F B(w) \cap \overline{B_{1 / 2}}$ satisfies

$$
\theta(r) \leq C \sigma(r)
$$


for a constant $C$.

Proof. Theorem (2.10) implies that by changing coordinates, if necessary, we can find a sequence $\left\{s_{k}\right\} \rightarrow 0$ such that our standard rescalings satisfy

$$
w_{s_{k}}(x):=\frac{w\left(s_{k} x\right)}{s_{k}^{2}} \rightarrow \frac{\left(x_{n}^{+}\right)^{2}}{2},
$$

where the convergence is uniform in $C^{1}$. The oscillation of the Laplacian of $w_{s_{k}}$ on $\bar{B}_{1}$ is bounded by $2 \sigma\left(s_{k}\right)$, and so by choosing $k$ sufficiently large we can be sure to satisfy the hypotheses of Theorem (5.4). Now let $w=$ $O P(\operatorname{Lap}=f, \operatorname{Bdry}=\psi)$, and then define $f_{k}(x):=f\left(s_{k} x\right)$ for $x \in \bar{B}_{1}$, and $\psi_{k}(x):=s_{k}^{-2} w\left(s_{k} x\right)$ for $x \in \partial B_{1}$. Then

$$
w_{s_{k}}(x)=O P\left(\operatorname{Lap}=f_{k}, \text { Bdry }=\psi_{k}\right),
$$

and if $l_{k}(x):=O P\left(\operatorname{Lap}=1+\sigma\left(s_{k}\right)\right.$, Bdry $\left.=\psi_{k}\right)$ and $h_{k}(x):=O P(\operatorname{Lap}=1-$ $\sigma\left(s_{k}\right)$, Bdry $\left.=\psi_{k}\right)$, then

$$
l_{k} \leq w_{s_{k}} \leq h_{k} .
$$

But now by Theorem (5.4) and Lemma (5.3) we know that the Hausdorff distance from $F B\left(w_{s_{k}}\right) \cap \overline{B_{1 / 2}}$ to $\left\{x_{n}=0\right\} \cap \overline{B_{1 / 2}}$ is bounded by $C \sigma\left(s_{k}\right)$. Scaling will now yield the desired conclusion.

Q.E.D.

Now we come to the main results of this paper.

7.2 Theorem ( Dini Laplacian Gives $\mathbf{C}^{1}$ Regularity ). Suppose $w \in$ $\widehat{\mathcal{P}}\left(\lambda, \mu, C^{\sigma}\left(\bar{B}_{1}\right)\right)$, and $\sigma$ satisfies the Dini condition:

$$
\int_{0}^{1} \frac{\sigma(r)}{r} d r<\infty .
$$

Then $F B(w)$ is $C^{1}$ in a neighborhood of the origin, and the modulus of continuity, $\zeta(s)$, of the derivative of the local parametrization is given by

$$
\zeta(s)=C \int_{0}^{s} \frac{\sigma(r)}{r} d r .
$$

In particular, if $\sigma \in C^{\alpha}$, then at regular points $F B(w)$ is $C^{1, \alpha}$ with the same $\alpha$.

Proof. The previous theorem immediately gives

$$
\int_{0}^{1} \frac{\theta(r)}{r} d r<\infty,
$$

and then Theorem (6.7) yields the desired result. Q.E.D. 
7.3 Theorem (Counter-Example). If $w \in \widehat{\mathcal{P}}\left(\lambda, \mu, C^{\sigma}\left(\bar{B}_{1}\right)\right)$, and $\sigma$ does not satisfy the Dini condition (7.3) above, then $F B(w)$ does not even have to be locally the graph of a function. In other words, we can find boundary data and a $\sigma$ for which the Dini integral diverges as slowly as we wish, such that the corresponding solution has a zero set with density one half at the origin, and whose free boundary is not even locally the graph of a function.

7.4 Remark. When one considers what we know about $w$, the fact that $F B(w)$ is not $C^{1}$ is quite surprising. We know the following:

- In appropriate coordinates, we know there is a sequence $\left\{s_{k}\right\}$ such that the rescalings $w_{s_{k}}$ converge in $C^{1, \alpha}\left(\bar{B}_{1}\right)$ to $\left(x_{n}^{+}\right)^{2}$. (See Theorem (2.10).)

- $F B(w)$ is a Reifenberg vanishing set in a neighborhood of the origin (see Theorem (6.4)), and we know the rate at which its width vanishes. (See Theorem (7.1).)

$$
\text { - } \quad \lim _{r \rightarrow 0} \frac{\left|\Lambda(w) \cap B_{r}\right|}{\left|B_{r}\right|}=\frac{1}{2} .
$$

(See Theorem (4.3).)

Proof. We will prove this theorem by explicitly creating a counter-example in two dimensions. We start with the following notation: $y$ will denote the usual "vertical" coordinate, and $y^{\prime}$ will be a very slight rotation of $y$. We will start with a function which is close to a signed distance function, and then we will square its positive part. First of all, let $\eta$ be a function which satisfies $0 \leq \eta \leq 1, \eta \in C_{0}^{\infty}\left(B_{2}\right)$, and $\eta \equiv 1$ in $B_{1}$. Now we define $d$ as follows:

On $R_{1}:=B_{1} \quad$ we let $d:=y^{\prime}$.

On $R_{2}:=B_{2}-B_{1}$ we let $d:=\eta y^{\prime}+(1-\eta) y$.

On $R_{3}:=B_{4}-B_{2}$ we let $d:=y$.

Since $d \in C^{\infty}$ and the vertical derivative of $d$ is at least $3 / 4$ we know that $\{d=0\}$ is a $C^{\infty}$ manifold by the Implicit Function Theorem.

So we have the following picture: 


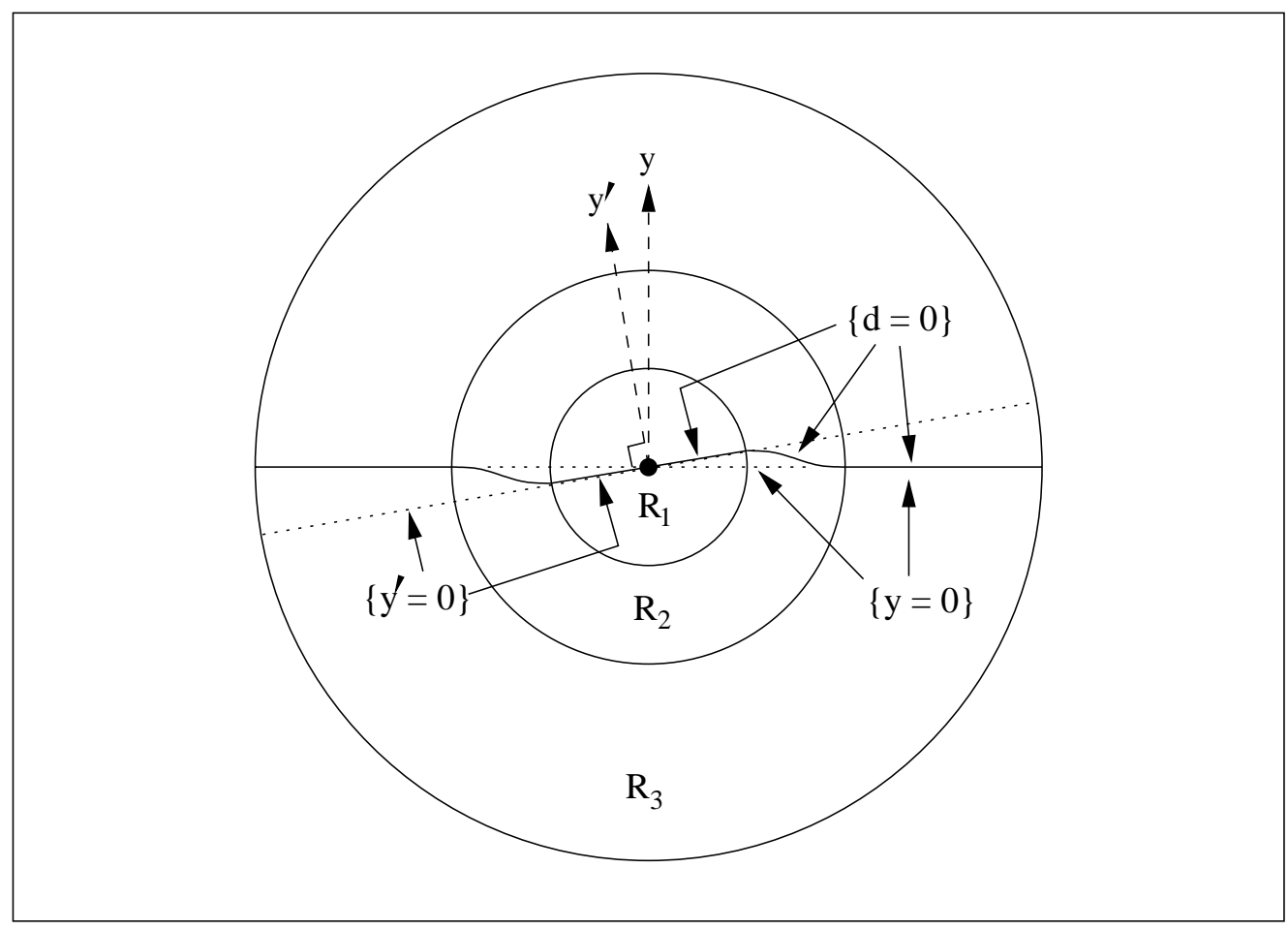

Now we redefine $d$ to be zero wherever it was negative, and we let

$$
w:=\frac{d^{2}}{2}
$$

On the set $\Omega:=\{w>0\}$ we have

$$
\Delta w=d \Delta d+\|\nabla d\|^{2} .
$$

Now note that (restricting our attention to $\Omega$ henceforth) in $R_{1}$ and in $R_{3}$, we have $\Delta d \equiv 0$ and $\|\nabla d\| \equiv 1$. So in those regions we have $\Delta w \equiv 1$. By our choice of $\eta$ we have $w \in C_{0}^{\infty}\left(\bar{B}_{4}\right)$. We need to compute $\Delta w$ in $R_{2}$ :

$$
\begin{gathered}
\frac{\partial d}{\partial x_{i}}=\frac{\partial}{\partial x_{i}}\left(\eta y^{\prime}+(1-\eta) y\right) \\
=\eta_{i}\left(y^{\prime}-y\right)+\eta\left(y_{i}^{\prime}-y_{i}\right)+y_{i} \\
\nabla d=\nabla \eta\left(y^{\prime}-y\right)+\eta\left(\nabla y^{\prime}-\nabla y\right)+\nabla y \\
\frac{\partial^{2} d}{\partial x_{i}^{2}}=\eta_{i i}\left(y^{\prime}-y\right)+2 \eta_{i}\left(y_{i}^{\prime}-y_{i}\right) \\
\Delta d=\left(y^{\prime}-y\right) \Delta \eta+2\left(\nabla y^{\prime}-\nabla y\right) \cdot \nabla \eta
\end{gathered}
$$




$$
\begin{aligned}
\|\nabla d\|^{2}= & \|\nabla \eta\|^{2}\left(y^{\prime}-y\right)^{2}+2 \eta\left(y^{\prime}-y\right)\left(\nabla \eta \cdot \nabla y^{\prime}\right) \\
& -2 \eta\left(y^{\prime}-y\right)(\nabla \eta \cdot \nabla y)+2\left(y^{\prime}-y\right)(\nabla \eta \cdot \nabla y) \\
& +2 \eta\left(\nabla y^{\prime} \cdot \nabla y\right)-2 \eta\|\nabla y\|^{2}+\|\nabla y\|^{2} \\
& +\eta^{2}\left(\left\|\nabla y^{\prime}\right\|^{2}-2\left(\nabla y^{\prime} \cdot \nabla y\right)+\|\nabla y\|^{2}\right) \\
= & 1+\|\nabla \eta\|^{2}\left(y^{\prime}-y\right)^{2}+2 \eta(\eta-1)\left(1-\nabla y^{\prime} \cdot \nabla y\right) \\
& +2\left(y^{\prime}-y\right)\left[(1-\eta)(\nabla \eta \cdot \nabla y)+\eta\left(\nabla \eta \cdot \nabla y^{\prime}\right)\right]
\end{aligned}
$$

Now if $\measuredangle\left(y^{\prime}, y\right)$ is sufficiently small, then

$$
|\Delta w-1|=|d \Delta d+||\nabla d|^{2}-1 \mid \leq 1 / 2,
$$

so $w \in \widehat{\mathcal{P}}_{4}\left(\lambda, \mu, C^{\sigma}\left(\bar{B}_{4}\right)\right)$. Of course, $F B(w)$ is smooth, so we are not done.

Now the plan is to repeat this process on smaller and smaller scales. In other words, we let $y^{\prime \prime}$ be a slight rotation of $y^{\prime}$ and start redefining $w$ on $B_{1 / 2}$. With $w:=[\max (d, 0)]^{2} / 2$ as before, we let $d$ be the same as before outside of $B_{1 / 2}$, but now we set $d:=y^{\prime \prime}$ on $B_{1 / 4}$, and $d:=\eta_{4} y^{\prime \prime}+\left(1-\eta_{4}\right) y^{\prime}$ on $B_{1 / 2}-B_{1 / 4}$, where $\eta_{4}(x):=\eta(4 x)$. Now we have $\Delta w \equiv 1$ on $B_{1 / 4}, B_{1}-B_{1 / 2}$, and $B_{4}-B_{2}$. So we keep repeating this process with the rotations $y^{(k)}$, and in every other annular region we will have $\Delta w \equiv 1 . w$ is $C^{\infty}$ outside of 0 , but we need control at 0 . We claim that in the $k^{\text {th }}$ nonlinear region

$$
\operatorname{osc}(\Delta w) \leq C \measuredangle\left(y^{(k)}, y^{(k-1)}\right)
$$

with a constant $C$ that does not depend on the scale. (In fact, $C$ depends on nothing.) In particular, if

$$
\lim _{k \rightarrow \infty} \measuredangle\left(y^{(k)}, y^{(k-1)}\right)=0
$$

then $\Delta w$ will be continuous at 0 . By taking a harmonic series of rotations, we will have an infinite rotation of $F B(w)$ around 0 . It remains to prove the claim. The idea is that although the scaling will make the derivatives of $\eta$ worse and worse, as we zoom in on 0 , the coordinate functions are getting smaller and smaller.

By combining our equations which started with equation (7.7), we can 
derive:

$$
\begin{aligned}
\Delta w-1 & =\|\nabla \eta\|^{2}\left(y^{\prime}-y\right)^{2}+2 \eta(\eta-1)\left(1-\nabla y^{\prime} \cdot \nabla y\right) \\
& +2\left(y^{\prime}-y\right)\left[(1-\eta)(\nabla \eta \cdot \nabla y)+\eta\left(\nabla \eta \cdot \nabla y^{\prime}\right)\right] \\
& +\left[\eta y^{\prime}+(1-\eta) y\right] \cdot\left[\left(y^{\prime}-y\right) \Delta \eta+2\left(\nabla y^{\prime}-\nabla y\right) \cdot \nabla \eta\right]
\end{aligned}
$$

Although this expression looks nasty, it is actually easy to see that its magnitude is unchanged by scaling, and that it vanishes as $\measuredangle\left(y^{\prime}, y\right)$ vanishes. We rewrite it with an overbrace (two overbraces) over terms which will have a factor of $r^{-1}\left(r^{-2}\right)$ with the rescaling to radius $r$, and an underbrace (two underbraces) under terms which will have a factor of $r\left(r^{2}\right)$ with the rescaling to radius $r$. We will box the terms which disappear as $\measuredangle\left(y^{\prime}, y\right) \rightarrow 0$.

$$
\begin{aligned}
& \Delta w-1=\overbrace{\|\nabla \eta\|^{2}}^{\underbrace{\left(y^{\prime}-y\right)^{2}}}+2 \eta(\eta-1) \underbrace{(1-\nabla y)}_{\left(1-\nabla y^{\prime} \cdot \nabla y\right)} \\
& +2 \underbrace{\left(y^{\prime}-y\right)}\left[(1-\eta)(\overbrace{\nabla \eta}^{\nabla \eta} \cdot \nabla y)+\eta(\overbrace{\nabla \eta}^{\nabla} \cdot \nabla y^{\prime})\right] \\
& +[\eta \underbrace{y^{\prime}}+(1-\eta) \underbrace{y}] \cdot[\underbrace{\left(y^{\prime}-y\right)} \overbrace{\Delta \eta}^{\Delta \eta}+2 \overbrace{\left(\nabla y^{\prime}-\nabla y\right)} \cdot \overbrace{\nabla \eta}]
\end{aligned}
$$

Inspection reveals that the scaling effects will cancel, so all that remains is to show that each boxed term is bounded by a constant times $\theta:=\measuredangle\left(y^{\prime}, y\right)$. But, since we have dealt with the scaling, it suffices to assume that the " $y$ 's" are of order 1. At that point trigonometry and Taylor series imply

$$
y^{\prime}-y \leq C \theta, \quad \nabla y^{\prime}-\nabla y \leq C \theta, \text { and } 1-\nabla y^{\prime} \cdot \nabla y \leq C \theta^{2} .
$$

Q.E.D.

7.5 Remark. This counter-example can also be used to show that the statement about the Hölder case given in Theorem (7.2) above is sharp.

7.6 Remark. All of the results in this section are trivial to extend to the nonlinear obstacle problem, as long as the operator satisfies the Caffarelli Alternative. 


\section{Acknowledgements}

I would like to thank The University of Texas for its hospitality while the bulk of this paper was written. This research was partially conducted by the author for the Clay Mathematics Institute. I would also like to thank Leonardo Bonorino, Tom Chunders, Ki-Ahm Lee, Jana Thompson, Peiyong Wang, Mike Webb, and Yu Yuan for stimulating conversations and for their support.

Most of all, I would like to express my deepest gratitude to my advisor, Professor Caffarelli.

\section{References}

[A] H.W. Alt, The fluid flow through porous media. Regularity of the free surface, Manuscripta Math., 21(1977), no. 3, 255-272.

[AC] I. Athanasopoulos and L.A. Caffarelli, A theorem of real analysis and its application to free boundary problems, Comm. Pure Appl. Math., 38(1985), no. 5, 499-502.

[BK] H. Brézis and D. Kinderlehrer, The smoothness of solutions to nonlinear variational inequalities, Indiana Univ. Math. J., 23(1973/74), 831-844.

[C1] L.A. Caffarelli, The regularity of free boundaries in higher dimensions, Acta Math., 139(1977), no. 3-4, 155-184.

[C2] L.A. Caffarelli, Compactness methods in free boundary problems, Comm. Partial Differential Equations, 5(1980), no. 4, 427-448.

[C3] L.A. Caffarelli, A remark on the Hausdorff measure of a free boundary, and the convergence of coincidence sets, Boll. Un. Mat. Ital. A, (5)18(1981), no. 1, 109-113.

[C4] L.A. Caffarelli, The obstacle problem revisited, J. Fourier Anal. Appl., 4(1998), no. 4-5, 383-402.

[CC] X. Cabré and L.A. Caffarelli, Fully Nonlinear Elliptic Equations, Amer. Math. Soc., 1995.

[CFMS] L.A. Caffarelli, E.B. Fabes, S. Mortola, and S. Salsa, Boundary behavior of nonnegative solutions of elliptic operators in divergence form, Indiana Univ. Math. J., 30(1981), no. 4, 621-640.

[CK] L.A. Caffarelli and D. Kinderlehrer, Potential methods in variational inequalities, J. Analyse Math., 37(1980), 285-295.

[F] J. Frehse, On the regularity of the solution of a second order variational inequality, Boll. Un. Mat. Ital., (4)6(1972), 312-315.

[GH] D. Gilbarg and L. Hörmander, Intermediate Schauder estimates, Arch. Ration. Mech. Anal., 74(1980), no. 4, 297-318.

[GT] D. Gilbarg and N.S. Trudinger, Elliptic Partial Differential Equations of Second Order, 2nd ed., Springer-Verlag, 1983. 
[JK] D.S. Jerison and C.E. Kenig, Boundary behavior of harmonic functions in nontangentially accessible domains, Adv. in Math., 46(1982), no. 1, $80-147$.

[KT1] C.E. Kenig and T. Toro, Harmonic measure on locally flat domains, Duke Math. J., 87(1997), no. 3, 509-551.

[KT2] C.E. Kenig and T. Toro, Harmonic measures and Poisson Kernels, Ann. of Math., (2)150(1999), no. 2, 369-454.

[KN] D. Kinderlehrer and L. Nirenberg, Regularity in free boundary problems, Ann. Scuola Norm. Sup. Pisa Cl. Sci., (4)4(1977), no. 2, 373-391.

[L] Ki-ahm Lee, Obstacle problem for nonlinear $2^{\text {nd }}$-order elliptic operator, preprint.

[LS1] H. Lewy and G. Stampacchia, On the regularity of the solution of a variational inequality, Comm. Pure Appl. Math. 22(1969) 153-188.

[LS2] H. Lewy and G. Stampacchia, On the smoothness of superharmonics which solve a minimum problem, J. Analyse Math. 23(1970) 227-236.

[R] E.R. Reifenberg, Solution of the Plateau Problem for $m$-dimensional surfaces of varying topological type, Acta Math., 104(1960), 1-92.

[S1] D.G. Schaeffer, The capacitor problem, Indiana Univ. Math. J., 24 (1974/75), no. 12, 1143-1167.

[S2] D.G. Schaeffer, A stability theorem for the obstacle problem, Adv. in Math., 17(1975), no. 1, 34-47.

[S3] D.G. Schaeffer, Some examples of singularities in a free boundary, Ann. Scuola Norm. Sup. Pisa., 4(1977), 133-144.

[St] S. Stojanovic, Perturbation formula for regular free boundaries in elliptic and parabolic obstacle problems, SIAM J. Control Optim., 35(1997), no. 6, 2086-2100.

[T1] T. Toro, Geometric conditions and existence of bi-Lipschitz parameterizations, Duke Math. J., 77(1995), no. 1, 193-227.

[T2] T. Toro, Doubling and flatness: geometry of measures, Notices Amer. Math. Soc., 44(1997), no. 9, 1087-1094.

[Z] A. Zygmund, Trigonometric Series, 2nd ed., Vol. I., Cambridge University Press, 1959. 